Continuous monitoring of left ventricular function by epicardial 3-axis accelerometers

Per Steinar Halvorsen

The Interventional Centre

Oslo University Hospital, Rikshospitalet

Faculty Division of Clinical Medicine

University of Oslo

Oslo, April 2010 
(C) Per Steinar Halvorsen, 2010

Series of dissertations submitted to the Faculty of Medicine, University of Oslo No. 1024

ISBN 978-82-8072-364-2

All rights reserved. No part of this publication may be reproduced or transmitted, in any form or by any means, without permission.

Cover: Inger Sandved Anfinsen.

Printed in Norway: AiT e-dit AS.

Produced in co-operation with Unipub.

The thesis is produced by Unipub merely in connection with the thesis defence. Kindly direct all inquiries regarding the thesis to the copyright holder or the unit which grants the doctorate. 



\section{Contents}

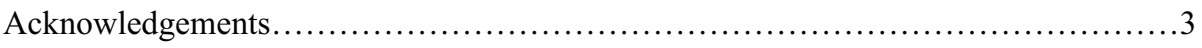

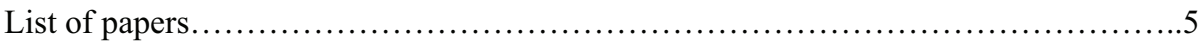

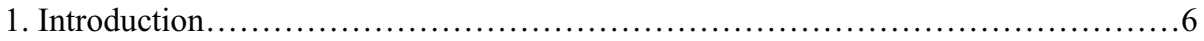

1.1 Motivation..........................................................

1.2 Left ventricular function.............................................6

1.3 Left ventricular contractions........................................ 7

1.4 Monitoring of regional ventricular function by echocardiography.............8

1.5 Monitoring of left ventricular function by 3 -axis accelerometer.............9

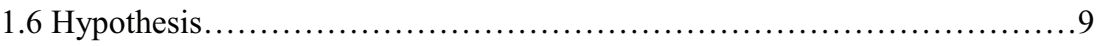

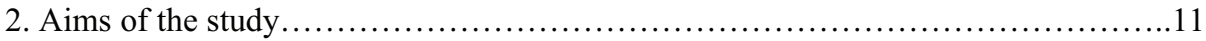

3. Material and methods .......................................................... 12

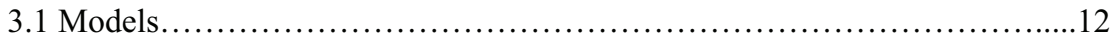

3.2 Accelerometer.................................................... 13

3.3 Echocardiography.................................................. 18

3.4 Hemodynamic measurements....................................... 19

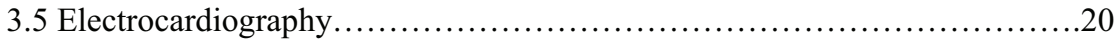

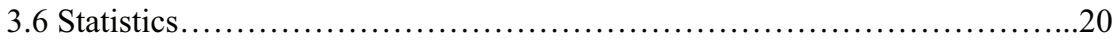

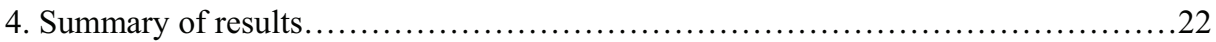

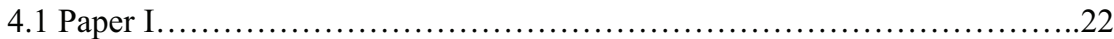

4.2 Paper II........................................................ 22

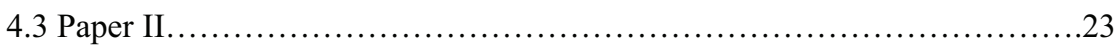

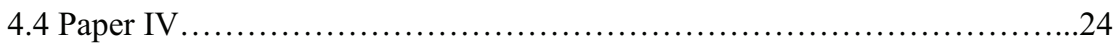

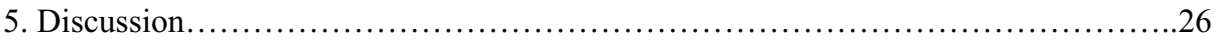

5.1 Detection of myocardial ischemia by 3 -axis accelerometer................26

5.2 Effects of tethering.................................................... 30

5.3 Accelerometer measurements and global left ventricular function..............31

5.4 Divergences in measured epicardial motions............................. 31

5.5 Accelerometer vs. other techniques................................ 32

5.6 Limitations..................................................... 32

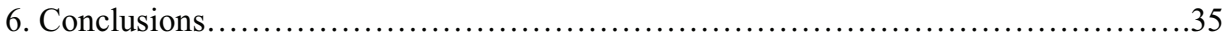

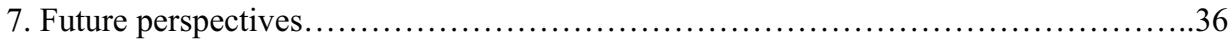

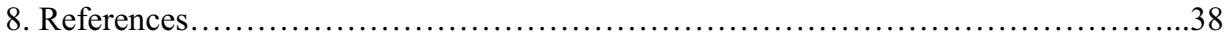

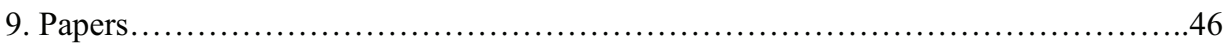




\section{Acknowledgements}

The present study was carried out during the years 2004 to 2009 at the Interventional Centre, Rikshospitalet, Oslo University Hospital, University of Oslo, Norway. The research has been supported by grants from The Regional Health Authorities of Southern Norway, Medinnova AS, Oslo University Hospital Rikshospitalet and from a clinical research fellowship from the Norwegian Council of Cardiovascular Diseases.

I would like to express my sincere gratitude to my three supervisors to whom I have been delighted to work with throughout these years. Despite his innumerable responsibilities as the head of the Interventional Centre at Rikshospitalet, my main supervisor professor Erik Fosse has been constantly supportive and enthusiastic. His bright ideas and knowledge of cardiovascular research have been essential in this thesis. I also want to acknowledge him for giving me the opportunity to work in an inspiring multidisciplinary environment. My second supervisor professor Tor Inge Tønnessen introduced me to the Interventional Centre and to experimental research. His experience and outstanding knowledge in this field and his curiosity of new concepts and ideas has been of great importance. Professor Halfdan Ihlen introduced me into the field of echocardiography and cardiac kinetics. As previous head of the echocardiography laboratory at Rikshospitalet and professor in echocardiography he has been an invaluable co-supervisor. He has always been available for encouraging academic discussions and has contributed substantially in the progress of the projects.

I am most grateful to my colleague and friend research fellow Andreas Espinoza. In the operating theatre and in our shared office we have had regular productive and unproductive discussions. He has been a key person and has contributed essentially in all studies included in this thesis. I want to express my thanks to Dr. Scient. Ole Jakob Elle in whom, together with professor Erik Fosse, had the idea of utilizing a 3-axis accelerometer for monitoring of myocardial function. With his background as an engineer he managed to build a bridge between the technical aspects and biological side of our research. The technical support from The Vestfold University Collage has been invaluable. Associated professor Kristin Imenes, associated professor Morten Grimnes and in particular research fellow Lars Albert Fleischer and associate professor Lars Hoff have all made essential contributions to the work in this thesis.

My sincere thanks go to the experienced staff of the Interventional Centre whose professionalism and positive spirit have been most valuable. In particular, I would like to thank thoracic surgeon Jacob Bergsland for superb cooperation, and Carmen Louwerens, Kjersti Wendt and Helga Teigland for their excellent assistance. 
Thanks to the Department of Cardiothoracic Surgery and to the highly experienced surgeons Runar Lundblad, Per Snorre Lingaas and Kjell Arne Rein, and to Helge Skulstad and Thor Edvardsen at the Department of Cardiology for their invaluable echocardiography assistance and encouraging discussions. I am also grateful to postdoctoral fellow Espen W. Remme for important contributions to the work in this thesis and to statistician Milada Cvancarova and to Harald Dugstad in Bio-Medisinsk Innovasjon AS for excellent support. Thanks to the Department of Anesthesiology and the Centre of Comparative medicine. Finally, special thanks to my children Eva and Olaf for their optimism and source of joy, and to my wife Helene for her love and support during these years.

Oslo, April 2010

Steinar Halvorsen 


\section{List of papers}

This thesis is based on the following papers, which will be referred to by their Roman numerals:

I. Elle OJ, Halvorsen S, Gulbrandsen MG, Aurdal L, Bakken A, Dugstad H, Fosse E. Early recognition of regional cardiac ischemia using a 3-axis accelerometer sensor. Physiol Meas. 2005;26:429-40.

II. Halvorsen PS, Fleischer LA, Espinoza A, Elle OJ, Hoff L, Skulstad H, Edvardsen T, Ihlen $\mathrm{H}$, Fosse E. Detection of myocardial ischaemia by epicardial accelerometers in the pig. Br J Anaesth. 2009;102:29-37.

III. Halvorsen PS, Espinoza A, Fleischer LA, Elle OJ, Hoff L, Lundblad R, Skulstad H, Edvardsen T, Ihlen H, Fosse E. Feasibility of a 3-axis epicardial accelerometer in detecting myocardial ischemia in cardiac surgery patients. J Thorac Cardiovasc Surg. 2008;136:1496-502.

IV. Halvorsen PS, Remme EW, Espinoza A, Skulstad H, Lundblad R, Bergsland J, Hoff L, Imenes K, Edvardsen T, Elle OJ, Fosse E. Automatic real-time detection of myocardial ischemia by epicardial accelerometer. J Thorac Cardiovasc Surg. 2010;139:1026-1032. 


\section{Introduction}

\subsection{Motivation}

In the last decade an increasing number of elderly patients with reduced left ventricular function are scheduled for cardiac surgery. ${ }^{1}$ Left ventricular function is directly linked to patients prognosis ${ }^{2}$ and it is therefore of vital importance to have sensitive and continuous methods for the assessment of left ventricular function. ${ }^{3}$ Major causes for reduced cardiac performance during surgery are global ventricular impairment due to relative hypovolemia (anesthetics, low intravascular volume, tamponade) $)^{4,5,6}$, reduced myocardial contraction as seen after weaning from extracorporeal circulation or from extensive myocardial ischemia. ${ }^{7}$ Persistent ischemia is associated with a tenfold increase in mortality and is therefore the most important factor for poor outcome. ${ }^{8}$ The occurrence of ischemia is followed by an immediate reduction in myocardial contraction. ${ }^{9}$ However, regional myocardial ischemia may not influence global left ventricular function, because adjacent regions that are not ischemic may compensate with increased contraction, preserving global left ventricular performance. ${ }^{10,11}$ In other cases, in particular in patients with extensive coronary artery disease, regional ischemia could lead to de-compensation of neighboring regions because of increased work load. This may eventually lead to global left ventricular collapse. ${ }^{12,13}$ The risk of myocardial infarction increases with the total load and duration of the ischemic events, ${ }^{14}$ and it is well recognized that prevention or treatment of ischemia leads to reduced perioperative cardiac morbidity. ${ }^{15,16}$ This includes maintaining hemodynamic stability, sufficient coronary artery perfusion pressure and avoidance of tachycardia. ${ }^{15,16,17}$ It is therefore of great importance that perioperative ischemia is detected and treated quickly in order to reduce postoperative cardiac complications. ${ }^{18,19}$

\subsection{Left ventricular function}

Systole begins with the onset of depolarization and contraction and ends at termination of contraction. Systolic ventricular function can be defined as the ventricle's capability to contract and eject blood against an afterload, and thus involves properties of the ventricle as a hemodynamic pump and the interrelated aspect on myocardial properties. ${ }^{20}$ Systolic myocardial function describes the degree of myocardial shortening which is determined by 1 ) muscle length at onset of contraction (preload), 2) contractility of the muscle, 3) afterload, which could be defined as the force against which myocardium contracts, 4) heart rate and rhythm. Myocardial contractility is a dynamic intrinsic characteristic of the tissue which is not influenced by pre-and afterload. Global contractility represents the performance of the left 
ventricle whereas regional contractility signifies the performance of a region of myocardium at a given preload and afterload. Changes in global contractility during cardiac surgery is typically seen during inotropic stimulation, whereas reduced regional myocardial contractility can frequently be observed due to coronary hypoperfusion and myocardial ischemia. ${ }^{21}$

The different end systolic pressure-volume relations form a linear relationship, and the slope of this line is called end systolic elastance. This parameter is an index of global left ventricular contractility and is nearly independent of loading conditions. ${ }^{22}$ The area within the pressure-volume loop describes myocardial work and has been proposed as a measure of elastance. ${ }^{23}$ However, these methods are not used clinically. The peak first derivate of systolic ventricular pressure, i.e. dp/dtmax, is an index of ventricular function which to some extent has been used clinically. ${ }^{24}$ Other clinical methods are ejection fraction and end systolic volume and derivates of these parameters. These parameters can be obtained by noninvasive echocardiography. They are, however, pre-and afterload dependent and mainly describe the pumping capacity of the heart and to a lesser degree myocardial contractility. ${ }^{25,26}$

In cardiac surgery left ventricular systolic function is usually judged by mean arterial blood pressure (afterload and stroke volume), central venous pressure (preload), heart rate and cardiac output. ${ }^{10,27}$ These routine methods poorly measure global left ventricular contractility and show inadequate sensitivity in detecting and localizing regional left ventricular dysfunction. ${ }^{27}$

\subsection{Left ventricular contractions}

Normal left ventricular contraction is characterized by longitudinal and circumferential shortening and wall thickening. ${ }^{28,29}$ The myofibers of left ventricle configures helicoids with two spiral turns. ${ }^{30}$ Viewed from the left ventricular apex, apical rotation is in the counterclockwise direction, whereas basal rotation is clockwise. Thus, normal circumferential motion changes from counter-clockwise to clockwise in the equatorial level of the left ventricle. This implies that there is a gradual decrease in circumferential motions from apex to the mid ventricular level. The ventricular basis moves towards the apex due to contractions of longitudinal orientated subendocardial and subepicardial myocardial fibers ${ }^{31,32}$ The ventricular apex is essentially stationary in closed chest conditions, ${ }^{33}$ and the most dominating contraction in this region is in the circumferential direction. ${ }^{34,35}$ Radial dimension of the left ventricle is reduced by both circumferential and longitudinal shortening. ${ }^{36}$ In the subendocardial layer velocity of myocardial contraction during systole is much larger than in the subepicardial 
layer. ${ }^{36}$ Thus, radial motion on the epicardium due to thickening and thinning of the heart wall is considered small.

A total occlusion of a coronary artery will induce transmural myocardial ischemia depending on collateral flow from other coronary arteries. This is due to a mismatch between oxygen supply and consumption. In response to this the myocytes in the ischemic segments stops contracting in order to survive (hibernating myocardium). ${ }^{37}$ All left ventricular layers are affected during transmural ischemia, and thus the ischemic segments may become entire passive and paradox movement of an ischemic region can be observed. ${ }^{21}$

\subsection{Monitoring of regional left ventricular function by echocardiography}

The basis for ischemia detection with echocardiography is that coronary hypoperfusion is followed by an immediate reduction in heart wall contraction in the supply area of the affected artery. ${ }^{38,21}$ The method can therefore be used to diagnose which coronary artery is hypoperfused and to evaluate the extent of ischemia. ${ }^{39}$ Depending on the degree of collateral flow from other coronary arteries, acute coronary artery occlusion and/or increased work load may further aggravate regional dysfunction. ${ }^{40}$ With echocardiography myocardial ischemia can be characterized as reduced and delayed systolic contraction or even paradox heart wall motion with post systolic contraction. ${ }^{41,42}$ Quantification of ischemia with echocardiography is usually not carried out during cardiac surgery, and the extent of ischemia is generally evaluated subjectively by visual assessment of the heart wall motion disturbances. ${ }^{39}$ In the last decade, new clinical echocardiographic methods have been developed. With assessment of myocardial strain (deformation) by tissue Doppler imaging, sensitive diagnosis of ischemia is possible. Additionally, a precise description and quantification of the affected area can be achieved. $^{41,43}$ Myocardial strain is caused by fiber contraction and thus is a measure of myocardial contractile function. Myocardial velocities and strains can be measured with echocardiography in all contraction directions intermittently by steering the ultrasonic beam. A reference of regional myocardial function is the left ventricle pressure-segment length (strain) loop which could be obtained with ultrasound techniques. ${ }^{44}$ The area within the loop described regional segmental work, and similar to the left ventricular systolic elastance, the end-systolic pressure segment length relationship has been proposed as a measure of regional elastance. ${ }^{23}$ Although providing very important clinical information these methods require complicated and time consuming off-line analysis. A skilled operator is needed to carry out the echocardiographic examination and only intermittent measurements are available. In addition, the technique is cumbersome to use in the postoperative period where myocardial 
ischemia and infarctions occur most frequently. ${ }^{39}$ Consequently, there is a need for new methods which can be used clinically for continuous monitoring of regional myocardial function and for detection of regional myocardial ischemia.

\subsection{Monitoring of left ventricular function by 3-axis accelerometer}

Sensors that measure acceleration (accelerometers) have been utilized in seismographs and navigation systems for many decades, but their use has increased considerably after silicon micromachining emerged in the 1990s. Currently accelerometers are included as sensors in consumer electronics, such as mobile telephones, portable media players, digital cameras and game consoles. In medicine accelerometers are mainly used for measuring physical activity, ${ }^{45}$ but can also be found in implantable pacemakers for the detection and treatment of arrhythmias. ${ }^{46,47}$

Single axis accelerometers have been used in a few previous studies to examine the origin of heart sounds and for investigation whether heart wall accelerations reflect ventricular contractility. ${ }^{48,46,49,50}$ A more recent investigation evaluated the use of an endocardial accelerometer for the detection of acute myocardial ischemia during percutaneous coronary intervention treatment. ${ }^{47}$ The main findings in these studies were that systolic accelerations correlated with the positive time derivate of ventricular pressure during inotropic stimulation, and that systolic accelerations decreased during temporary coronary artery occlusion. These results therefore indicate that accelerometers can be used for measuring ventricular function. In the previous clinical studies, assessing ventricular function by accelerometer, the sensor was incorporated into a pacemaker lead and positioned on the endocardium in the right ventricular apex. ${ }^{46,47}$ The intention for clinical use of accelerometers in these studies was limited to patients requiring permanent pacemaker treatment.

\subsection{Hypothesis}

The idea to use miniaturized 3-axis accelerometer for early operator independent detection of myocardial ischemia during and after cardiac surgery was initiated by the Interventional Centre at Rikshospitalet in 2001. With the use of a 3-axis accelerometer ventricular contraction could be automatically and continuously assessed in three directions. The intention was to incorporate the sensor into temporary pacemaker leads which could be attached to the epicardium during surgery and withdrawn in the postoperative period when risk of ischemia is not longer imminent. The overall hypothesis was that this combined pacemaker and accelerometer lead could improve the diagnosis of myocardial ischemia, 
which is a prerequisite for treatments that can reduce morbidity and mortality in cardiac surgery.

Being the first study on the use of accelerometer in the clinical setting of cardiac surgery, the primary goal in this investigation was to test the performance of the accelerometer in ischemia detection. It was therefore hypothesized that the 3-axis accelerometer could detect and quantify regional left ventricular dysfunction induced by temporary coronary artery occlusion (paper I) with similar sensitivity and specificity as echocardiography (paper II-IV) and with higher sensitivity and specificity than routine methods used during cardiac surgery (paper II-IV). It was also hypothesized that epicardial contractions in longitudinal, circumferential and radial directions measured with the 3 -axis accelerometer are equally affected during coronary artery occlusion (paper I-III) and that the 3-axis accelerometer discloses the same information about myocardial contraction as echocardiography during normal and ischemic conditions (paper II-IV). Finally, it was hypothesized that operator independent automated detection of myocardial ischemia is possible using epicardial acceleration signals (paper IV).

The hypothesis was tested in experimental and clinical models using an open prospective repeated measurement design. Experimentally left descending coronary artery was temporary occluded in a pig model to test sensitivity of the sensor in ischemia detection (paper I, II and part of paper IV), whereas in paper II and part of paper IV pharmacologic interventions were made to change global ventricular function to test specificity of the sensor. The 3-axis accelerometer was validated for ischemia detection in the clinical setting on patients undergoing off-pump coronary artery bypass grafting during left anterior descending coronary artery occlusion (paper III and part of paper IV). 


\section{Aims of the study}

To validate a 3-axis epicardial accelerometer for continuous assessment of left ventricular function in normal and ischemic myocardium:

1. by investigating whether 3-axis accelerometers could be used for continuous detection of regional left ventricular dysfunction during temporary coronary artery occlusion,

2. by comparing the quantitative and qualitative changes in epicardial contractions in longitudinal, circumferential and radial directions to the corresponding myocardial strains obtained with echocardiography during temporary coronary artery occlusion,

3. by comparing changes in epicardial contractions to the corresponding hemodynamic and electrocardiographic changes during temporary coronary artery occlusion,

4. by investigating whether epicardial motions measured with the 3-axis accelerometer are markers of global left ventricular function during pharmacologic interventions by correlating the epicardial motions to known experimental and clinical parameters of ventricular function,

5. by testing sensitivity and specificity of algorithms for automated ischemia detection with the 3-axis accelerometer during reduced myocardial function induced by temporary coronary artery occlusion and by pharmacologically induced $\beta$-blockade. 


\section{Material and methods}

A total of 17 pigs were included in the experimental studies, 3 in study I and 14 in study II. Ten of the 14 pigs included in study II were also analyzed in the experimental part of study IV. Two pigs died before data collection was completed, thus in this study data from 15 animals are presented. Ten patients were included in the clinical material. Of the 10 patients included in study III, seven were also included in the clinical part of study IV, because in these patients left ventricular dysfunction was observed with echocardiography during coronary artery occlusion.

\subsection{Models}

The 3-axis accelerometer was tested experimentally and clinically in an open chest and open pericardium model.

\subsubsection{Experimental model}

Initial tests on performance of the 3-axis accelerometer were carried out on pigs (paper I, II and part of paper IV). All pigs were treated according to The 1996 Guide for the Care and Use of Laboratory Animals. ${ }^{51}$ Anesthesia and surgical preparation are described in details in paper II. In the experimental studies one 3-axis accelerometer was sutured on the apical anterior wall of the left ventricle. This region is perfused by the left anterior descending coronary artery and was used as the interventional region in all studies. The apical anterior wall of the left ventricle was chosen for study because this region is most frequently grafted during coronary artery bypass surgery. In addition, the anatomy of the coronary arteries allowed more reproducible placement of the accelerometer from animal to animal. To examine the capability of the sensor in detecting regional changes in left ventricular function during coronary artery occlusion an additional 3-axis accelerometer was sutured in the perfusion region of the circumflex coronary artery (control sensor). The pigs remained placed in the supine position with the chest and the pericardium left open.

In all experimental studies the animals were their own controls. Baseline recordings were performed after a stabilizing period of at least 30 minutes. The left anterior descending coronary artery was temporary occluded for one (paper I and II), three (paper IV) and five minutes (paper I) to test sensitivity of the sensor and for measuring which epicardial contraction direction is most affected during coronary artery occlusion. Accelerometer measurements were also obtained after occlusion for evaluating the restoration of left 
ventricular function during reperfusion (paper I and II). For testing specificity of the sensor global left ventricular function was changed by interventions altering preload, afterload and contractility (nitroprusside, esmolol, epinephrine and fluid loading) in paper II, whereas in paper IV a global reduction left ventricular function was induced by esmolol. Invasive measurements (except paper 1) and echocardiography were used as reference techniques for detecting changes in hemodynamics and myocardial function. Electrocardiography (except study 1) and echocardiography were used for diagnosing myocardial ischemia.

\subsubsection{Clinical model}

The performance of the accelerometer was tested clinically on patients undergoing off pump coronary artery bypass grafting in paper III and IV. Anesthesia and surgery are described in details in paper III. This model allowed investigating sensitivity and specificity of the sensor in detecting myocardial ischemia almost in an experimental way. Similar as in the experimental model one 3-axis accelerometer was positioned on the apical anterior region of the left ventricle. Regional myocardial dysfunction was induced by a three minutes pregrafting occlusion of the left anterior descending coronary artery followed by a five minutes reperfusion period. After completed revascularization, but before chest closure, the internal mammary artery graft to the left anterior descending coronary artery was occluded for three minutes to investigate specificity of the accelerometer and to test whether graft occlusion was possible to detect with the 3-axis accelerometer. Invasive measurements, electrocardiography and transoesophageal echocardiography were used as reference techniques as described for the experimental model.

\subsection{Accelerometer}

Two 2-axis accelerometers (ADXL-202, Analog Devices Inc, Noorwood, MA, USA) were mounted perpendicular to each other in order to make a 3-axis acquisition of the acceleration data in paper I, whereas a specialized 3-axis accelerometer (Kionix Inc, KXM52-1050, Itacha, NY, USA) (paper IV, Figure 1) consisting of three piezoelectric plates assembled $90^{\circ}$ to each other were used in paper II-IV. Movement of the sensor induces bending of the piezoelectric plates by inertial forces and is the principle of sensing and measuring acceleration with the sensor. This causes changes in conductivity of the plates which eventually can be sensed and quantified. Mass of the sensor with silicon coating was $0.8 \mathrm{~g}$. Measurement range was \pm 2 of the gravitational field $(\mathrm{G})$ and cross-sensitivity between axes was $2 \%{ }^{52}$ The gravitational field was used for calibrating the accelerometer. All acceleration signals were recorded 
synchronously with pressures and electrocardiography at a sampling rate of 250 to $500 \mathrm{~Hz}$. The acceleration signals were high pass filtered in order to reduce noise from the $220 \mathrm{~V}$ net $(50 \mathrm{~Hz})$ and to remove fluctuations in the signals due to effects from ventilation.

\subsubsection{Measurement of epicardial motion directions}

The cardiac coordinate system was used to assess left ventricular wall motions with echocardiography. The 3-axis accelerometer was aligned to this system in paper I-IV to compare the epicardial motions obtained with the accelerometer with the correspondent myocardial contractions measured with echocardiography. The accelerometer $\mathrm{x}$-axis paralleled the longitudinal-, y-axis the circumferential- and z-axis the radial left ventricular contraction directions. The acceleration signals were analyzed separately in the longitudinal, circumferential and radial directions in paper I-III to test the hypothesis that the epicardial contractions are equally affected during coronary occlusion.

\subsubsection{Timing of heart phases}

Myocardial ischemia induced by coronary artery occlusion is characterized with regional decreased early systolic contraction and increased early diastolic contraction. In early diastole, the isovolumic relaxation phase is particularly affected. Contraction in this phase is considered a marker of myocardial ischemia and is widely used. ${ }^{53,38}$ The changes in wall motions within these different heart phases during coronary artery occlusion can be precisely detected and quantified with echocardiography. ${ }^{41,54}$ In order to measure left ventricular epicardial motions in both early systole and diastole these heart phases had to be identified accurately. This was achieved by gating the acceleration signals to electrocardiography (paper II-IV) and to left atrial and ventricular pressures (paper II). Systole was defined from peak R on electrocardiography to the peak negative time derivate of left ventricular pressure (dP/dtneg). Diastole was defined from the peak negative time derivate of left ventricular pressure to $\mathrm{R}$ on electrocardiography. The isovolumic relaxation phase was defined within the period from the peak negative time derivate of left ventricular pressure to the cross-over of left atrial- and left ventricular pressures. This enabled examining whether the 3-axis accelerometer disclose the same information on heart motions in these phases as echocardiography, both during normal and pathological left ventricular contraction.

In the clinical setting left ventricular pressure is not available and timing of the isovolumic relaxation phase had to be performed differently. This heart phase has previously been accurately determined using myocardial acceleration/time curves, in which aortic valve 
closure and mitral valve opening were automatically identified as distinct spikes. $^{55,56,57}$ Therefore, to overcome this problem and for evaluating the accelerometers in the clinical setting, the accelerometer signals were used to determine this heart phase (paper III).

During systole longitudinal acceleration was defined positive from basis to apex, circumferential acceleration was defined positive in the counter clockwise direction and radial acceleration was defined positive towards the left ventricular lumen.

\subsubsection{Parameters measured from the 3-axis accelerometer}

Myocardial acceleration, velocity and displacement are measures of cardiac function. ${ }^{58,59,60,61}$ By measuring one of these, the two others may be derived by integration or derivation, e.g. velocity and displacement may be calculated from the time integral and double time integral, respectively, of a measured acceleration signal. ${ }^{62}$ Thus, with the accelerometer it is possible to measure all these parameters. Velocity and displacement are well known parameters for measuring myocardial function with echocardiography, ${ }^{38,59}$ whereas myocardial accelerations are less examined. A reason for that could be that the interpretation of myocardial accelerations can be difficult in the clinical setting, because opening and closure of heart valves may generate large accelerations that do not necessarily reflect myocardial contraction. ${ }^{62,46,50}$ To overcome this potential problem, and for comparisons with echocardiography, epicardial velocities and displacements were also calculated from the accelerometer signals.

\section{Analysis on raw acceleration signals}

In paper I the hypothesis was tested using diverse analyses of the raw acceleration signals in longitudinal, circumferential and radial directions. Peak positive systolic acceleration and the difference between peak positive and negative systolic acceleration were measured, since previous investigations suggest that these parameters can be used for measuring ventricular performance. ${ }^{47}$

Changes in higher frequencies of the acceleration signals were also analyzed in paper I, as myocardial ischemia has been found to induce great effects on the distribution of these frequencies. ${ }^{50}$ There is an analogy between sound and acceleration. Heart wall vibrations are generated when the myocardium contracts isometrically and from opening and closing of valves and blood flowing through the heart and great vessels. ${ }^{63,64,65}$ These vibrations are responsible for the heart sounds and can be easily heard using a stethoscope. Both the audible and inaudible spectrum of these vibrations can be measured with an epicardial 
accelerometer. ${ }^{56}$ The tone level changes according to changes in frequency, however, the same tone played by different instruments may sound very different due to differences in the strength (or power) of the higher frequencies. If one considers the epicardium a loudspeaker membrane and the accelerometer the microphone, the heart rate or resonant frequency of the heart is the "tone". The resonant frequencies depend on the stiffness and geometry of the vibrating ventricles, which could both be affected during coronary occlusion since ischemic segments behave like net passive structures. The principle of analyzing higher frequencies of epicardial acceleration signals has previously been used to examine the origin of heart sounds and for investigating effects of regional myocardial ischemia on the first heart sound. ${ }^{48,50}$ In these studies a single axis accelerometer was used to generate time-frequency transforms (spectrograms) of the acceleration signal in the radial direction. The higher frequencies of the acceleration signals, as measured in the spectrograms, were markedly changed in the ischemic region during coronary artery occlusion. In order to measure left ventricular dysfunction during coronary artery occlusion and to examine whether the epicardial accelerations were equally affected analyses of higher frequencies were therefore also performed on the acceleration signals obtained from the 3 -axis accelerometer.

Analyses of higher frequencies were made by two methods based on previous investigations. ${ }^{49,50} \mathrm{~A}$ detailed description of these methods can be found in paper I. In summary a short-time Fourier transform of the acceleration signals was performed to generate spectrograms with time on the x-axis and the different frequencies (higher harmonics) of the acceleration signals on the y-axis. In the spectrogram the first harmonic indicates the heart rate (analog to sound; the tone level). Power of the different frequencies of the acceleration signals is indicated by color in the spectrogram, and red specify the strongest and blue the weakest power (paper I, Figure 3). The spectrograms were made to examine the changes in this frequency pattern during coronary artery occlusion. In order to quantify these changes the difference in power spectrum of the acceleration signal ( $\Delta$ SignalPower) was computed (paper I equation 1, Figure 5). In contrast to previous studies, examining signal frequency distribution within a specific time interval in systole, we examined signal changes within the entire heart cycle. The reason for doing that was that the changes in higher frequencies during coronary artery occlusion might be even more evident, since both systolic and diastolic functions are affected during ischemia. To allow such analysis more than one heart cycle was included in the sliding reference window.

In addition, to separate analysis of contractions in longitudinal, circumferential and radial directions, the epicardial acceleration vector length $\left(\sqrt{ } x^{2}+y^{2}+z^{2}\right)$ was also examined 
by the methods described. The reason for doing that was that this vector is independent on orientation of the sensor on the heart surface.

\section{Analysis of epicardial velocities}

The acceleration signals were numerically integrated to obtain epicardial velocities in the longitudinal, circumferential and radial directions for comparisons with echocardiography. ${ }^{66}$ In the cardiac cycle peak early systolic ejection velocity in addition to the largest velocity spike during the isovolumic relaxation are sensitive in the detection of myocardial ischemia when measured with the ultrasound technique. Left ventricular dysfunction during coronary artery occlusion is characterized with a decrease in peak early systolic ejection velocity together with an increase in velocity during the isovolumic relaxation phase, ${ }^{41}$ and therefore velocities in similar heart phases were also measured with the 3-axis accelerometer.

\section{Analysis of epicardial displacements}

Epicardial displacements were calculated from the accelerometer by double numeric integration of the acceleration signals. The rational for doing this was to examine which accelerometer parameter was most sensitive in detecting myocardial ischemia. Additionally, a standard method for measuring regional left ventricular work experimentally is the left ventricular-myocardial segment length (-strain) loop area. ${ }^{44,67,68}$ To further evaluate the accelerometer in ischemia detection left ventricular pressure-epicardial displacement loops were generated (paper IV). The area within the loops was used as surrogate of regional myocardial work, similar to the left ventricular pressure-segment length loop obtained with echocardiography. The objectives were to investigate whether the accelerometer generated loop area was a sensitive measure of regional myocardial dysfunction, and whether ischemia induced similar changes in the accelerometer generated loops as previously described in left ventricular pressure-segment length loop obtained with the ultrasound technique.

\section{Automated analysis}

For automated ischemia detection with the accelerometer and for investigating which accelerometer parameter was most sensitive, peak systolic velocity and displacements within a 150 millisecond interval after the electrocardiography R-peak were automatically measured. These analyses were based on the knowledge that cardiac wall motions in early systole is greatly affected during coronary artery occlusion $^{38,24}$ and that defining end-systole and 
detecting the isovolumic relaxation phase in the clinical setting might be difficult, while detection of systole using the electrocardiography R-peak is more feasible.

\subsection{Echocardiography}

In paper II-IV echocardiography was used as reference for the detection of left ventricular dysfunction during coronary artery occlusion and for comparing myocardial motions obtained with this technique with corresponding epicardial motions measured with the 3-axis accelerometer.

The echocardiographic examinations were obtained by a Vivid 7 scanner (GE Vingmed, Horten, Norway). In the experimental studies, the ultrasound probe was placed directly on the heart using an ultrasound gel stand-off to obtain high quality images. The gel allowed the heart wall to glide under the ultrasound probe with as little as possible influence on left ventricular contraction. The technique has been used in several previous experimental studies to evaluate left ventricular function. In the clinical studies the echocardiographic images were obtained with a transesophageal probe. This method has been extensively evaluated clinically and has demonstrated excellent ability for monitoring left ventricular function during coronary artery occlusion. ${ }^{35,69,40}$

Conventional two-dimensional grayscale echocardiography images were obtained from left ventricle short axis at two left ventricle levels, through the apical and basal regions in both the experimental and clinical studies. Analyses were performed off-line with the software program EchoPAC (GE Vingmed Ultrasound, Horten, Norway).

\section{Two-dimensional strain echocardiography}

Myocardial strains were measured in paper II-IV. Myocardial strains reflect left ventricular systolic function more precisely than myocardial velocities measured with tissue Doppler echocardiography, since strains are less sensitive to segment tethering (motions in one segment are determined by function in other neighboring segments as well) and cardiac translation (movement that changes the position of an object, i.e motion from the entire heart moving).

Strain defines fractional tissue deformation in response to applied force (stress). In the myocardium this is directly related to myocardial fiber shortening. ${ }^{70,68,71}$ Thus, strain represents fractional change of tissue length, i.e. changes in length per unit length. If an object has an initial length $\mathrm{L}_{0}$, and after a certain time changes to the length $\mathrm{L}$, strain is defined as 
Strain $=\frac{\mathrm{L}-\mathrm{L}_{0}}{\mathrm{~L}_{0}}$

Strain reflects deformation of the myocardium and thus describes the contraction/relaxation pattern. Myocardial strain is expressed as percent shortening or lengthening, depending on which direction is being measured. By convention, in the longitudinal and circumferential directions, negative strain is defined as shortening and positive strain as lengthening, whereas in the radial direction positive strain is defined as thickening.

Myocardial strains were calculated from ultrasound speckles based on grayscale images (two-dimensional strain). ${ }^{72}$ The method is semi-automatic and tracks the speckle pattern seen in the grayscale images during one cardiac cycle. The region of interest is marked manually and throughout the cardiac cycle, peak systolic strain is calculated as a percentage of end-diastolic dimensions. End-systole was defined after peak T, whereas end-diastole was defined at start $\mathrm{R}$ in the electrocardiogram. An advantage of measuring strain with the speckle tracking method compared to the tissue Doppler techniques is that accuracy of the speckle tracking method is not dependent on the angle between the myocardium and the ultrasonic beam. $^{73} \mathrm{~A}$ decline in systolic strain is a marker of reduced regional systolic myocardial function and is used as a direct sign that ischemia occurs during the coronary occlusions..$^{73,72}$

Myocardial strains in the apical anterior and mid lateral regions of the left ventricle were assessed for comparisons with the corresponding epicardial motions measured with the 3-axis accelerometers. Thus, peak systolic strains in the longitudinal, circumferential and radial directions were measured in both regions. The mean of three consecutive heart cycles was used for statistical calculations.

\subsection{Hemodynamic measurements}

\subsubsection{Pressures}

Left ventricular pressure was obtained with a 5 Fr Millar micro manometer-tipped catheter. Based on previous experimental studies the peak positive time derivate of left ventricular pressure $(\mathrm{dP} / \mathrm{dtmax})$ was used as a marker of global ventricular contractility in the animal studies (paper II and IV). ${ }^{74,75}$ The left ventricular pressure was also used for generating left ventricular pressure-epicardial displacement loops as a measure of regional myocardial work. Detailed descriptions can be found in paper II and IV. 
An objective in this study was to validate the accelerometer measurements against cardiovascular monitoring used routinely in cardiac surgery. Thus, in a clinical study (paper III) mean arterial blood pressure and its positive time derivate ( $\mathrm{dP} / \mathrm{dtmax})$ and central venous pressures were measured.

\subsubsection{Flow}

Cardiac output describes the pumping capacity of the heart at a given preload and afterload. ${ }^{20}$ The parameter is widely used clinically as a marker of cardiac function ${ }^{76}$ and was therefore included in this study to describe and quantify the hemodynamic changes induced by the different interventions, and for examining whether the accelerometer measurements correlated to the changes in cardiac output. Experimentally (paper II), cardiac output was measured as flow in aorta ascendence by using a $16 \mathrm{~mm}$ ultrasonic flow probe (Medistim, Oslo, Norway). Aortic flow was averaged over a time interval of 7 seconds to obtain reliable measurements. In patients cardiac output was measured with pulse-contour analyses using a PiCCO monitor (versions 5.1, Pulsion Medical, Munchen, Germany) (paper III and IV). With this method stroke volume is calculated from the systolic portion of the femoral arterial pressure curve. The method has been validated during cardiac surgery in several previous studies. ${ }^{77,78,79}$ The rational for using the technique in this study was that cardiac output could be measured continuously in real time. Thus, any change in left ventricular pumping capacity induced by coronary artery occlusion could potentially be detected almost immediately.

\subsection{Electrocardiography}

Analysis of the ST-segment in the electrocardiography signals are routinely used clinically to detect regional left ventricular myocardial ischemia. ${ }^{39,14}$ This analysis was therefore also included in both experimental and clinical studies for comparisons with the accelerometer measurements. In this study the ST-segment was defined 80 milliseconds from the J-point and standard electrocardiography lead II was monitored in order to detect apical anterior left ventricular ischemia. ST-segment depression greater than $0.1 \mathrm{mV}$ was considered ischemia.

\subsection{Statistics}

In all papers parametric statistical analysis was performed and data are presented as mean \pm standard deviation of the sample distribution. A repeated measurement design was used in all papers. Multiple paired sample t-tests were used for the repeated measurements in paper II, III and clinical part of paper IV, and included all baselines and the corresponding interventional 
values. In order to evaluate whether the experimental model vas hemodynamically stable during the experiments, a repeated measurement analysis of variance was performed on all baseline values in paper II. This analysis was also performed in the experimental part of paper IV, on both baseline and interventional values. Bonferroni corrections of the P-values were made in all papers and a $\mathrm{P} \leq 0.05$ was considered significant. The Pearson correlation coefficient was calculated in order to describe the association between accelerometer measurements and hemodynamic and echocardiographic parameters, and together with the Bland-Altman method, ${ }^{80}$ to describe reproducibility of the accelerometer measurements. Receiver operating characteristic curves were generated in paper II, III and IV to calculate cut-off values for ischemic states and to estimate sensitivity and specificity of the accelerometer methods for detecting ischemia. Positive and negative predictive values of the accelerometer measurements were computed from table analysis, and the $95 \%$ confidence intervals constructed with the f-distribution. 


\section{Summary of results}

\subsection{Paper I}

Early recognition of regional cardiac ischemia using a 3-axis accelerometer sensor. Physiol Meas. 2005;26:429-40.

The aim of this experimental pilot study on three pigs was to test the ability of the 3-axis accelerometer in detecting regional left ventricular dysfunction induced by coronary artery occlusion by analyses of raw acceleration signals. The study demonstrated the feasibility of monitoring myocardial dysfunction by the 3-axis accelerometer. Significant and almost instant changes were observed in all contraction directions during left anterior descending coronary artery occlusion. This was found by measuring positive and negative peak systolic accelerations and by use of methods for analyzing the higher frequency distribution of the acceleration signals. The study also established that the accelerometer vector length, which includes accelerations in longitudinal, circumferential and radial directions, could be used as a measure of left ventricular function. For this vector the positive early systolic acceleration was reduced by from $0.352 \mathrm{G}$ to $0.075 \mathrm{G}$ during coronary occlusion, while the negative early systolic acceleration changed from $0.55 \mathrm{G}$ to $0.45 \mathrm{G}$. The difference between positive and negative peak systolic accelerations decreased by approximately $60 \%$ during two minutes occlusion of left descending coronary artery. Another main finding in this study was that the epicardial accelerations were unequally affected during coronary artery occlusions. The accelerometer y-axis, measuring epicardial acceleration in the circumferential direction, was most affected in all pigs. This was evident when using analysis of higher frequencies of the accelerometer signal. Immediate and large increases in power of higher harmonics occurred in the spectrograms during ischemia. The study also demonstrated that these changes were possible to quantify by using the difference value between the actual power spectrum and a baseline power spectrum.

\subsection{Paper II}

Detection of myocardial ischaemia by epicardial accelerometers in the pig. Br J Anaesth. 2009; 102:29-37.

In this study the main aim was to test sensitivity and specificity of the 3 -axis accelerometer in detection regional left ventricular dysfunction induced by coronary artery occlusion. For comparisons with echocardiography and for evaluating epicardial motions in detecting myocardial ischemia, velocities in longitudinal, circumferential and radial directions were calculated from the 3-axis accelerometer signals. Two sensors were sutured on the left 
ventricle, one in of the supply area of left descending coronary artery (interventional region) and the other in a control region (circumflex supplied area). Regional left ventricular dysfunction was confirmed with strain echocardiography in the interventional region, but not in the control region during one minute of left descending coronary artery occlusion. Both during normal and ischemic conditions, the accelerometer generated epicardial velocity curves looked similar to those obtained with the ultrasound method. The traces were of high quality with low intra- and inter observer variation. In the interventional region peak early systolic velocity in the circumferential direction decreased significantly $(14.1 \pm 3.4$ to $6.0 \pm$ $2.5 \mathrm{~cm} / \mathrm{s}$ ) during occlusion, whereas velocity in the isovolumic relaxation phase increased $(-5.3 \pm 3.3$ to $7.7 \pm 6.6 \mathrm{~cm} / \mathrm{s})$. Similar, but non-significant changes were also found in longitudinal and radial directions during ischemia. These results demonstrated the ability of the accelerometer to detect left ventricular dysfunction during coronary artery occlusion, and confirmed the findings in paper I that epicardial contraction in the circumferential direction was most affected during occlusion. In contrast to the echocardiography strain measurements, significant changes were also found in accelerometer velocities in the control region during occlusion. Thus, the 3-axis accelerometer showed less ability than strain echocardiography to precisely localize regional dysfunction. This study also documented that epicardial peak systolic velocity in the circumferential direction was a measure of global left ventricular function by correlating to changes in the positive time derivate of left ventricular pressure $(r=0.81)$ and cardiac output $(r=0.73)$ during interventions affecting global left ventricular function (nitroprusside, esmolol, epinephrine and fluid loading). Even though the interventions on global left ventricular function caused the greatest hemodynamic changes, the accelerometer was capable in distinguishing left ventricular dysfunction induced by coronary artery occlusion from alterations in global function induced by the pharmacologic interventions. The sensitivity and specificity of the accelerometer in detecting ischemia by circumferential peak systolic and isovolumic relaxation velocities ranged from $94-100 \%$ and from $92-94 \%$, respectively. Another main finding was that the accelerometer was superior to electrocardiography ST-segment monitoring, since there was no significant change in this parameter during coronary occlusion.

\subsection{Paper III}

Feasibility of a 3-axis epicardial accelerometer in detecting myocardial ischemia in cardiac surgery patients. J Thorac Cardiovascular Surg. 2008; 136:1496-502 
This study aimed to test the 3-axis accelerometer in continuous detection of myocardial ischemia during cardiac surgery. In 10 patients undergoing off-pump coronary artery bypass grafting, one 3-axis accelerometer was sutured on the left ventricle in the supply region of left descending coronary artery. Temporary occlusions (3 minutes) of the left descending coronary artery at start of surgery and the left internal thoracic artery at end of surgery were performed to test sensitivity and specificity of the sensor. A total of 20 occlusions were investigated. Of them 7 occlusions of left descending coronary artery induced apical anterior left ventricular dysfunction when measured with the reference method (strain echocardiography). A main finding in this study was that during the occlusions where left ventricular dysfunction was found by echocardiography there were no significant electrocardiographic or hemodynamic changes, while significant changes were observed in accelerometer circumferential peak systolic $(\mathrm{P}<0.01)$ and isovolumic velocities $(\mathrm{P}<0.05)$. Thus, the accelerometer was superior to routine methods used for continuous detection of myocardial ischemia during cardiac surgery. The epicardial velocities by the 3-axis accelerometer were unequally affected during coronary artery occlusion in patients. Similar to the experimental studies the most significant changes were observed in the circumferential direction. In contrast, myocardial strains as measured by echocardiography were significantly changed in all contraction directions during ischemia. This implies that there is a distinction between the methods in assessing heart wall motions and that epicardial motions may be more affected by translational forces from non-ischemic neighboring regions. Another major finding was that during the 13 occlusions that regional left ventricular dysfunction was not observed by echocardiography, no dysfunction was found with the accelerometer. Consequently, the accelerometer demonstrated similar ability as echocardiography to detect ischemia in patients.

\subsection{Paper IV}

Automatic real time detection of myocardial ischemia by epicardial accelerometer. J Thorac Cardiovascular Surg. In press.

The main aim in this study, including 10 pigs and 7 patients, was to test algorithms for automated real-time detection of left ventricular dysfunction by accelerometer measurements in both experimental and clinical settings. One accelerometer was fixed to the epicardium in the area perfused by left descending coronary artery. Peak circumferential velocity and displacement were automatically calculated from the acceleration signals within 150 milliseconds after peak R on electrocardiography. Additionally, in the experimental part of the study, left ventricular pressure-epicardial displacement loop area was used as a measure of 
regional left ventricular work. Global left ventricular function was altered by esmolol infusion and regional dysfunction induced by temporary left descending coronary artery occlusion. Of the accelerometer measurements, mid systolic displacement demonstrated the greatest change during the coronary artery occlusion in both the experimental and clinical settings. Although the greatest hemodynamic changes occurred during the intervention with esmolol, the automated accelerometer methods enabled discrimination of regional left ventricular dysfunction induced by coronary artery occlusion from a global reduction induced by esmolol with sensitivity and specificity of $100 \%$. In the clinical material the automated accelerometer methods detected ischemia in 6 of 7 patients (sensitivity of $86 \%$ ). Another finding in the study was that mid systolic displacement correlated to the left ventricular pressure-epicardial displacement loop area $(\mathrm{r}=0.90)$, indicating that this parameter may be used as a surrogate for regional work. Finally, it was found that mid systolic displacement changed even more than the loop area during coronary occlusion. This demonstrated that the reduction in epicardial contraction during ischemia primarily occurred during early systole. 


\section{Discussion}

\subsection{Detection of myocardial ischemia by 3-axis accelerometer}

In these studies we demonstrated that an epicardial 3-axis accelerometer enables earlier and more sensitive diagnosis of left ventricular dysfunction caused by coronary artery occlusion than hemodynamic and electrocardiographic ST-segment monitoring, and that the method offer a possibility for continuous automated monitoring of left ventricular ischemia very similar to what can be periodically obtained by echocardiography. The 3-axis accelerometer was able to detect and quantify left ventricular dysfunction with comparable sensitivity and specificity as echocardiography (paper II -IV) and the epicardial motions reflected myocardial motions measured with the ultrasound technique, both in normal and ischemic conditions (paper II-IV) All epicardial contraction directions were affected by coronary artery occlusion, however, the dysfunction in apical left ventricular contraction was most pronounced in the circumferential direction. This was found both experimentally and clinically and was most likely due to transmural ischemia, affecting the dominating left ventricular circumferential orientated myofibers (paper I-IV). ${ }^{81,36}$

\subsubsection{Frequency analysis}

Coronary artery occlusion induced immediate and large alterations in the epicardial acceleration signals frequency distribution, confirming results from previous studies using single axis accelerometers. ${ }^{50}$ These results demonstrate that it is possible to detect and quantify left ventricular dysfunction continuously with the accelerometer. However, pilot experiments performed prior to study II showed that interventions affecting global left ventricular function also induced great changes in acceleration signal frequency distribution. These changes were difficult to differentiate from changes induced by coronary artery occlusion. Therefore, apparently sensitive for detecting ischemia, these methods may show poor specificity in recognizing ischemia. It might have been easier to characterize ischemia by analyzing changes in the frequency distribution within specific heart phase rather than analyzing changes from heart beat to heart beat, similar to what was done in the study by Wood and co-workers. ${ }^{50}$ In this study median frequency distribution in early systole was increased $31 \%$ within 5 minutes of coronary occlusion. However, likewise in paper I coronary artery occlusion was the only interventions to induce changes in left ventricular performance in this study, thus specificity of such analysis has not been tested. Additionally, the change in frequency was much less compared to the approximately $200 \%$ changes in the calculated difference value described in Figure 4 and 5 in paper I. Supplementary analysis of frequency 
distribution of the epicardial acceleration signals are therefore subjected to further studies in our laboratory.

\subsubsection{Peak systolic accelerations}

In paper I a large decline in peak systolic epicardial accelerations was found during coronary artery occlusion, demonstrating that epicardial accelerations are markers of regional myocardial contractility. This result is in accordance with a previous study by Theres and coworkers. ${ }^{47}$ They examined endocardial peak systolic accelerations for ischemia detection during per cutaneous coronary interventions. In our study coronary artery occlusion induced a much larger effect on systolic accelerations compared to the study by Theres and co-workers; a reduction of $\sim 40 \%$ in epicardial acceleration vs. $7 \%$ decline in endocardial acceleration, respectively. This may be explained by differences between species regarding the extent of collateral flow and the degree of coronary artery disease, but also that different ventricles were investigated. Nevertheless, the results from paper I indicate that epicardial accelerations have similar ability to detect left ventricular dysfunction as endocardial accelerations.

Peak systolic acceleration is caused by isovolumetric contraction. ${ }^{60,61}$ In the normal ventricle isovolumetric contraction can be defined as segmental shortening before onset of ventricular ejection, thus is active myocardial contraction in start systole. However, mitral valve closure may also induce ventricular vibrations, ${ }^{62,46}$ which can be seen as multiple spikes in the epicardial acceleration signals in both late diastole and early systole. These accelerations can be difficult to separate from accelerations caused by ventricular contraction and therefore epicardial accelerations could be difficult to interpret in the clinical setting. In addition, by use of sonomicrometry it has been found that isovolumetric accelerations were less affected than ejection velocities during coronary artery occlusion. ${ }^{62} \mathrm{~A}$ more detailed analysis of epicardial motions was therefore performed by analysis of accelerometer velocities in paper II-IV and displacements in paper IV in order to find the most sensitive parameter for ischemia detection.

\subsubsection{Epicardial velocities}

The results on epicardial velocities demonstrate that it is possible to detect left ventricular dysfunction induced by coronary artery occlusion with high sensitivity (paper I-IV) and specificity (paper II-IV) by use of accelerometer technology. Under normal conditions the epicardial velocity traces looked similar to myocardial velocity traces obtained with echocardiography. ${ }^{41}$ There was a short positive velocity spike during the isovolumic 
contraction phase in all accelerometer velocity traces. This was followed by positive velocities during ejection and negative velocities in the isovolumic relaxation phase. In diastole early filling due to mitral valve opening (E') and late filling by atrial contraction (A') were detectable in all directions as distinct negative velocity spikes (paper IV, Figure 2). Thus, the epicardial 3-axis accelerometer provides information on left ventricular systolic and diastolic function comparable to tissue Doppler imaging.

The epicardial velocity curves were characterized by reduced systolic velocities and concurrent increases in isovolumic relaxation velocities during coronary artery occlusion. Thus, the changes in epicardial velocities were qualitatively similar to changes seen in myocardial velocities measured by sonomicrometry and tissue Doppler imaging. ${ }^{38,41,54}$ In the experimental studies, both systolic and isovolumic relaxation velocities changed much more during coronary artery occlusion than during interventions affecting global left ventricular function, even though the interventions on global function induced greater hemodynamic changes. The results also indicates that epicardial ejection velocities are more sensitive parameters of left ventricular function than epicardial peak systolic accelerations, since greater changes were observed in ejection velocities during occlusion.

An interesting finding in this study was that epicardial ejection velocities in longitudinal, circumferential and radial directions differed to some extent. The longitudinal ejection velocity was dominated by an early large velocity spike which was followed by almost zero or even negative velocities in late systole. In contrast, circumferential and radial ejections velocities were positive and relative constant during the whole systole. This might be explained by different activation of longitudinal and circumferential fibers during systole. ${ }^{81,36}$ Left ventricle systolic contraction is initiated by contraction of longitudinal oriented fibers and is followed by activation of the dominating circumferential oriented fibers. ${ }^{36}$ Thus, it is likely that the accelerometer epicardial velocities reflect this contraction pattern. The open chest open pericardium model may explain the negative late systolic velocities in the longitudinal direction, as apical left ventricular longitudinal motion may be reversed under these circumstances. ${ }^{40}$

\subsubsection{Epicardial displacements}

Displacements reflect the sum of all velocities in a time interval and thereby potentially provide more information. In paper IV, both experimentally and clinically, the greatest change in accelerometer measurements during coronary artery occlusion was found by automated analysis of epicardial early systolic displacement. This indicates that displacement is the most 
sensitive accelerometer parameter for monitoring left ventricular dysfunction. This result is in accordance with previous findings using echocardiography, showing that systolic displacement performed better than ejection velocities in detecting ischemia and that this parameter was closest correlated to myocardial segmental work. ${ }^{59}$ The accelerometer displacement curves looked qualitatively similar to myocardial displacement curves obtained with echocardiography (paper IV, Figure 2). ${ }^{59}$ Therefore, the left ventricular pressureaccelerometer displacement loops looked similar to left ventricular pressure-segment length loops generated by sonomicrometry or echocardiography. ${ }^{44}$ This implies that the accelerometer provides the same information on the relationship between left ventricular pressure and left ventricular motions during systole and diastole. In normal conditions there is a great increase in pressure during the isovolumic contraction phase, while almost no epicardial displacement occurs (paper IV, Figure 3). In contrast, during ejection epicardial displacement is large despite little change in pressure. The loop is reversed during diastole (isovolumic relaxation and filling).

In paper IV it was also found that the left ventricular pressure-accelerometer displacement loops looked qualitatively similar to loops generated with ultrasound techniques, both in normal and ischemic conditions. The loop area and early systolic displacement were significantly more reduced during coronary artery occlusion than during the intervention with esmolol. Thus, the accelerometer displacements discriminated between regional and global left ventricular dysfunction.

Another important finding on epicardial displacement in paper IV was that early systolic displacement was even more changed than the left ventricular pressure-accelerometer displacement loop area. This indicates that the reduction in epicardial contraction during ischemia primarily occurs during early systole. In contrast, Skulstad and co-workers showed that myocardial displacement by sonomicrometry was found reduced during whole systole during comparable duration of coronary artery occlusion in dogs. ${ }^{59}$ Compared to myocardial displacement by sonomicrometry the epicardial systolic contractions therefore seem to be more affected by translation heart motion. This was considered when developing algorithms for automated analysis of accelerometer signals, and therefore epicardial motions in early systole were measured by use of this analysis.

\subsubsection{Automated analysis of accelerometer signals}

Automated analysis of accelerometer signals may facilitate the use of accelerometers during and after cardiac surgery. This study clearly demonstrates that it was possible to detect 
regional left ventricular dysfunction induced by coronary artery occlusion with high sensitivity and specificity with use of automated analysis of accelerometer systolic velocity and displacement. This was evident also in patients undergoing coronary artery bypass surgery (paper IV), despite coronary artery occlusion induced no hemodynamic or electrocardiography changes. Such analysis of accelerometer signals may provide continuous operator independent early warning of myocardial ischemia. A premise for automated ischemia detection with accelerometers is that the signals are of high quality. This was demonstrated by our results regarding intra- and interobserver variation (paper II-III). In addition, exact timing of the accelerometer signals is important to obtain reliable and analyzable data. Our system enables real-time presentation of both accelerometer signals and electrocardiography. A robust timing of the accelerometer signals to systole was ensured by gating the signals to peak $\mathrm{R}$ on electrocardiography. Automated real-time ischemia detection by use of this algorithm depends on a high quality electrocardiography signal and accuracy of the method may be influenced by conduction system abnormalities, arrhythmias and heart rate changes. However, these limitations are also seen with echocardiography and electrocardiographic ST-segment monitoring. ${ }^{82}$

\subsection{Effects of tethering}

A characteristic with epicardial motions by accelerometer was that they were affected by function in other left ventricular segments as well. This is also seen when measuring myocardial motions with the tissue velocity imaging technique and is due to tethering between segments and cardiac translation motion. ${ }^{59}$ Reduced epicardial motions in a region may therefore not necessarily reflect a decreased function in these segments. This was documented in paper II as accelerometer velocities were significantly reduced in the nonischemic region, while the echocardiography strain values remained unchanged. Likewise contractions in non-ischemic regions may influence epicardial contraction velocities in neighboring ischemic regions due to tethering and cardiac translation. ${ }^{83}$ Therefore, similar to the tissue velocity technique, nonviable myocardium may appear to contract. This implies that the accelerometer can not be regarded as a strictly regional method for detecting ischemia and that myocardial strains by echocardiography were superior to the accelerometer in defining the anatomical extension of the myocardial dysfunction. However, due to effects of tethering the number of sensors needed for monitoring all coronary artery supply areas may be reduced.

Both experimentally and clinically myocardial strains changed to a greater extent than accelerometer measurements during coronary artery occlusion. This indicates that strains by 
echocardiography are more sensitive in ischemia detection. Nevertheless, the accelerometer has an obvious advantage in the clinical setting, since continuous detection of ischemia is possible, while intermittent measurements and off-line analysis complicates the use of speckle tracking echocardiography.

\subsection{Accelerometer measurements and global left ventricular function}

Accelerometers can also be used to monitor global ventricular performance. In the current study a strong correlation was demonstrated between accelerometer circumferential peak systolic velocity and the peak positive time derivate of left ventricular pressure and cardiac output during interventions affecting global left ventricular function. This denotes that the accelerometer had the ability to measure global left ventricular function and that this parameter may be used as a substitute for left ventricular contractility and pumping capacity. These results indicate that the accelerometer can be used in the guidance of effects of treatment and in hemodynamic stabilization of the patients. From a clinical point of view it is of great interest to distinguish ischemia from other causes affecting left ventricular function. Myocardial ischemia regularly occurs in connection with cardiac surgery and alterations in global left ventricular function are frequent due to changes in anesthesia, volume status, myocardial contractility and vascular resistance. In paper II and IV accelerometer velocities and displacements during ischemia were quantitatively and qualitatively different from the corresponding velocities obtained during interventions on global left ventricular function. Post systolic prominent positive velocities, as regularly are found during ischemia, were not observed during the interventions affecting global left ventricular function. We therefore concluded that the accelerometer had the ability to distinguish ischemia from changes in load and contractility. For hemodynamic and electrocardiographic monitoring this is not possible, and as a potential monitoring device during cardiac surgery the accelerometer definitely has an advantage.

\subsection{Divergences in measured epicardial motions}

The accelerometer measurements reflected known left ventricular contraction pattern. In normal conditions left ventricular epicardial motions in interventional and control regions differed to some extent. Circumferential motion was largest in the interventional region, whereas longitudinal epicardial motion was greatest in the more basal control region (paper II). Radial motion was found similar in both regions. These results are in accordance with other studies using echocardiography and magnetic resonance imaging, showing that 
longitudinal shortening increases towards the basis of the heart and that circumferential motion changes from counter-clockwise to clockwise in the equatorial level of the left ventricle. ${ }^{33}$ This implies that there is a gradual decrease in circumferential motions from apex to the mid ventricular level. This also explains the finding in paper II that the accelerometer systolic circumferential velocities were found greatest in apical region of left ventricle.

\subsection{Accelerometer vs. other techniques}

Echocardiography, magnetic resonance imaging, isotope and radiological techniques have dominated assessment of left ventricular function in the clinical situation. In this work we have shown that the 3 -axis accelerometer is a promising future tool. Besides the accelerometer method epicardial motions have also been measured continuously by use of video, ${ }^{84,85,86}$ electromagnetic coils ${ }^{87,88}$ and gyro sensors. ${ }^{89}$ These techniques have only been utilized experimentally in open chest models, and are most likely due to their complicated use in the clinical setting, and that the techniques are difficult to miniaturize. Furthermore, except for the gyro technique, more than one sensor or marker is needed for measuring motion. Continuous monitoring of regional left ventricular function is also possible with implantable miniature ultrasonic crystals (sonomicrometry), ${ }^{38,23,90}$ but this method is not suitable in clinical practice due to its invasiveness. Recently, we successfully tested a miniature epicardial ultrasound transducer for detection of regional myocardial function by continuous assessment of transmural myocardial velocities. ${ }^{91}$ Miniature ultrasonic transducers may therefore become an alternative to the accelerometers in continuous perioperative monitoring. However, accuracy of this method depends on correct angel of the ultrasonic beam, and more sensors are probably required for monitoring left ventricle since the method is strictly regional. Further studies are needed to determine which sensor is most suitable in cardiac surgery.

\subsection{Limitations}

In this thesis we used a model of coronary artery occlusion which resulted in great changes in left ventricular function, shown by the strain echocardiography measurements. Our accelerometer results were therefore obtained during severe ischemia. The present findings and proposed use of epicardial accelerometers may not be applicable to subendocardial ischemia, which represent a significant fraction of ischemia in cardiac surgery. Moreover the duration of acute ischemia in patients varies widely and there may be considerable changes in intensity of ischemia over time. All our hypotheses have been tested under well controlled experimental conditions and our conclusions may be related to these situations. The clinical 
scenario is much more complex and further studies must be performed in order to examine whether the concept of ischemia detection with epicardial accelerometers is of value in patients, in particular in the postoperative phase. In this thesis coronary artery occlusion was only performed during open chest situations and the accelerometer measurements may not be valid in closed chest conditions. The regional epicardial response to coronary artery occlusion, especially in longitudinal direction, may be different. The presently large size of the prototype accelerometer did not allow investigating the effects of chest closure on the accelerometer measurements. However, we still believe that the model is valid for studying the performance of the sensor in ischemia detection.

No other methods for measuring epicardial motions were used in our model and therefore accuracy of the accelerometer measurements are uncertain. Left ventricular epicardial accelerations in longitudinal, circumferential and radial directions are expressed as vectors which are given by sum of the translatory and rotational components. An accelerometer can not distinguish between translatory acceleration and rotation in a gravitational field. ${ }^{66}$ This can be particularly relevant in the left ventricular apex since during systolic ejection epicardial apex rotation is found to be approximately $-11^{\circ}$. In general, rotation of the sensor in a gravitation field will be measured as increased motion by the accelerometer. The effects of ventricular rotation on the accelerometer measurements have recently been studied in detail and an algorithm for adjustment of rotation has been proposed..$^{92}$ Nevertheless, further studies must be undertaken in order to investigate the effects of rotation on the accelerometer measurements. In addition, the effects of change in body position have to be further investigated.

Another limitation is that ischemia was only introduced in the left descending coronary artery perfusion area. Further investigations should address to what extent accelerometer measurements are influenced by the presence of ischemia in other heart regions and to determine how many sensors are needed for monitoring ischemia in the presence of multi vessel disease.

In our model the duration of coronary artery occlusion was based on previous studies demonstrating left ventricular dysfunction almost immediately after coronary occlusion. The short occlusion time was sufficient to induce left ventricular dysfunction. However, it is likely that myocardial ischemia defined as myocardial anaerobic metabolism and lactacidosis was not present in our coronary artery occlusion model, since high energy phosphate components in the myocardium is not fully consumed after 1-3 minutes of coronary occlusion. ${ }^{93}$ The motion disturbances can persist for hours after prolonged occlusion despite reperfusion and 
restoration of myocardial energy balance. ${ }^{94}$ In this situation, known as myocardial stunning, the myocardium is viable..$^{95}$ Motion disturbances caused by stunning may be difficult to separate from dysfunction caused myocardial infarction when using accelerometers. However, this is also a limitation with the ultrasound technique. Further studies are needed in order to examine performance of the accelerometer during prolonged ischemia and to test the ability of the sensor in separating viable from nonviable myocardium. At present such a study is performed by our group with use of accelerometer and measurements of myocardial metabolism, such as lactate, glucose, tissue tension of carbon dioxide and oxygen.

The electrocardiography ST segment in lead II was only monitored in this study. It is likely that myocardial ischemia could have been detected with higher sensitivity with use of 5 or 12 lead electrocardiography. ${ }^{14}$ However, electrocardiography has generally shown much lower sensitivity and specificity in detecting perioperative ischemia than echocardiography. ${ }^{39}$ Echocardiography was therefore used as reference for evaluating the accelerometer in ischemia detection, and since 3 lead electrocardiography is most often used in cardiac surgery it was also utilized in this study.

Aortic flow might be affected by coronary artery occlusion. An alternative is to measure flow in arteria pulmonalis and this has been implemented in our present pig models. 


\section{Conclusions}

1. The 3-axis accelerometer can be used for continuous detection of myocardial ischemia. The technique showed almost similar ability as echocardiography to detect left ventricular dysfunction during coronary artery occlusion. Epicardially placed 3axis accelerometers offer a possibility for continuous monitoring of left ventricular motions very similar to what can be periodically obtained by echocardiography.

2. The 3-axis accelerometer detected regional ischemia with higher sensitivity and specificity than hemodynamic parameters and electrocardiography ST-segment monitoring.

3. Epicardial accelerations, velocities and displacements by accelerometer were parameters of regional left ventricular function. Coronary occlusion induced greatest effect on epicardial displacement.

4. Left ventricular epicardial contractions in longitudinal, circumferential and radial directions were quantitatively different. Coronary artery occlusion induced greatest dysfunction in the circumferential direction and systolic velocity in this direction was a marker of global left ventricular function during pharmacologic interventions.

5. Detection of left ventricular dysfunction was possible by automated analysis of epicardial accelerometer signals. The technique therefore offers promise as a potential monitor for ischemic events. 


\section{Future perspectives}

A monitoring system with a miniaturized 3-axis accelerometer incorporated into temporary pacemaker leads, together with automated analysis of signals may become important sensors for detection of myocardial ischemia and for monitoring left ventricular performance. Further miniaturization of the accelerometer is expected in the near future and at present a 3-axis accelerometer with dimensions of $2 \times 2 \times 0.95$ millimeter is commercial available (VTI Technologies CMA3000-A01, Helsinki, Finland). Based on results from this study we have developed a specialized software program for online analysis of signals. Like electrocardiography ST-segment analysis it gives the possibility to choose accelerometer axis, parameter and time interval for measurements. A chosen average (5-60 seconds) of accelerometer signals is continuously displayed on the monitor and compared to a baseline template. The cut-off values for the different accelerometer parameters could be implemented as indicators of ischemic state. The program also allows analyzing the signals as percentage change from a baseline signal. If there is a reduction $>30 \%$ in epicardial motion an alarm indicator shifts from green to yellow and further to red if motion is reduced $>50 \%$.

Further signal analysis may improve sensitivity of the sensor in ischemia detection. The 3 dimensional concept of the accelerometer was not completely investigated in this study. The program also provides monitoring the sum vector of the 3 accelerometer axis, as suggested in paper I. This vector is less dependent on orientation of the sensor to the cardiac coordinate system. By use of the $\mathrm{x}, \mathrm{y}, \mathrm{z}$ coordinates it may also be possible to automatically find and monitor the most dominating epicardial contraction, regardless placement of the sensor on the left ventricle. With the accelerometer it is also feasible to generate epicardial 3 dimensional displacements loops where 3 dimensional displacements can be measured as contraction length during systole and diastole. Pilot experiments have demonstrated the possibility of such analyses to detect ischemic motions as coronary artery occlusion induced a typically twist in the 3 dimensional displacement loop in addition to a great reduction in systolic length of contraction. No such twist was seen during interventions affecting global cardiac functions.

In addition to automatic ischemia detection the accelerometer parameters can be used as surrogates for contractility. The sensor may therefore also be important in monitoring and stabilizing of high risk patients with reduced left ventricular function. In particular the technique may be beneficial in monitoring graft function after heart transplantation. In the future we envision miniaturized wire less accelerometers may be implanted on the left ventricle in high risk patients during surgery. After hospital discharge such permanent sensors 
may give continuous information on heart rate, arrhythmias, ventricular performance and occurrence of ischemic events during daily activities. This offers promise for better diagnosis, earlier treatment of complications and improved guidance of interventions. 


\section{References}

1. Drury NE, Nashef SA. Outcomes of cardiac surgery in the elderly. Expert Rev Cardiovasc Ther 2006; 4(4): 535-542.

2. Swaminathan M, Morris RW, De Meyts DD, Podgoreanu MV, Jollis JG, Grocott HP, Milano CA, Newman MF, Mathew JP. Deterioration of regional wall motion immediately after coronary artery bypass graft surgery is associated with long-term major adverse cardiac events. Anesthesiology 2007; 107(5): 739-745.

3. Khalid MA, Bhatia A, Gal R. Echocardiographic Assessment of Complications Related to Left Ventricular Function in Acute Myocardial Infarction. Echocardiography 1999; 16(3): 281-287.

4. Kuvin JT, Harati NA, Pandian NG, Bojar RM, Khabbaz KR. Postoperative cardiac tamponade in the modern surgical era. Ann Thorac Surg 2002; 74(4): 1148-1153.

5. Reuter DA, Felbinger TW, Schmidt C, Moerstedt K, Kilger E, Lamm P, Goetz AE. Trendelenburg positioning after cardiac surgery: effects on intrathoracic blood volume index and cardiac performance. Eur J Anaesthesiol 2003; 20(1): 17-20.

6. Schmidt C, Roosens C, Struys M, Deryck YL, Van NG, Colardyn F, Van AH, Poelaert JI. Contractility in humans after coronary artery surgery. Anesthesiology 1999; 91(1): 58-70.

7. Doenst T, Borger MA, Weisel RD, Yau TM, Maganti M, Rao V. Relation between aortic cross-clamp time and mortality - not as straightforward as expected. Eur J Cardiothorac Surg 2008; 33(4): 660-665.

8. De Hert SG, Adriaensen HF. Perioperative myocardial ischaemia and infarction in connection with cardiac and non-cardiac surgery. Acta Chir Belg 1989; 89(2): 66-72.

9. Theroux P, Franklin D, Ross J, Jr., Kemper WS. Regional myocardial function during acute coronary artery occlusion and its modification by pharmacologic agents in the dog. Circ Res 1974; 35(6): 896-908.

10. Edgerton JR, Dewey TM, Magee MJ, Herbert MA, Prince SL, Jones KK, Mack MJ. Conversion in off-pump coronary artery bypass grafting: an analysis of predictors and outcomes. Ann Thorac Surg 2003; 76(4): 1138-1142.

11. Abraham TP, Nishimura RA, Holmes DR, Jr., Belohlavek M, Seward JB. Strain rate imaging for assessment of regional myocardial function: results from a clinical model of septal ablation. Circulation 2002; 105(12): 1403-1406.

12. Jacquet L, Hanique G, Glorieux D, Matte P, Goenen M. Analysis of the accuracy of continuous thermodilution cardiac output measurement. Comparison with intermittent thermodilution and Fick cardiac output measurement. Intensive Care Med 1996; 22(10): 1125-1129.

13. Lazor MA, Pierce ET, Stanley GD, Cass JL, Halpern EF, Bode RH. Evaluation of the accuracy and response time of STAT-mode continuous cardiac output. Journal of Cardiothoracic and Vascular Anesthesia 1997; 11(4): 432-436. 
14. Landesberg G, Mosseri M, Zahger D, Wolf Y, Perouansky M, Anner H, Drenger B, Hasin Y, Berlatzky Y, Weissman C. Myocardial infarction after vascular surgery: the role of prolonged stress-induced, ST depression-type ischemia. J Am Coll Cardiol 2001; 37(7): 1839-1845.

15. Slogoff S, Keats AS. Does perioperative myocardial ischemia lead to postoperative myocardial infarction? Anesthesiology 1985; 62(2): 107-114.

16. Slogoff S, Keats AS. Does chronic treatment with calcium entry blocking drugs reduce perioperative myocardial ischemia? Anesthesiology 1988; 68(5): 676-680.

17. Slogoff S, Keats AS, Ott E. Preoperative propranolol therapy and aortocoronary bypass operation. JAMA 1978; 240(14): 1487-1490.

18. Mangano DT, Browner WS, Hollenberg M, London MJ, Tubau JF, Tateo IM. Association of perioperative myocardial ischemia with cardiac morbidity and mortality in men undergoing noncardiac surgery. The Study of Perioperative Ischemia Research Group. N Engl J Med 1990; 323(26): 1781-1788.

19. Mangano DT, Siliciano D, Hollenberg M, Leung JM, Browner WS, Goehner P, Merrick S, Verrier E. Postoperative myocardial ischemia. Therapeutic trials using intensive analgesia following surgery. The Study of Perioperative Ischemia (SPI) Research Group. Anesthesiology 1992; 76(3): 342-353.

20. Levy MN. The cardiac and vascular factors that determine systemic blood flow. Circ Res 1979; 44(6): 739-747.

21. Theroux P, Ross J, Jr., Franklin D, Kemper WS, Sasyama S. Regional Myocardial function in the conscious dog during acute coronary occlusion and responses to morphine, propranolol, nitroglycerin, and lidocaine. Circulation 1976; 53(2): 302-314.

22. Burkhoff D, Mirsky I, Suga H. Assessment of systolic and diastolic ventricular properties via pressure-volume analysis: a guide for clinical, translational, and basic researchers. Am J Physiol Heart Circ Physiol 2005; 289(2): H501-H512.

23. Miller WP, Liedtke AJ, Nellis SH. Regional end-systolic pressure-length relationships using a volume-loading technique in the intact pig heart. Circ Res 1984; 55(3): 326335.

24. Quinones MA, Gaasch WH, Alexander JK. Influence of acute changes in preload, afterload, contractile state and heart rate on ejection and isovolumic indices of myocardial contractility in man. Circulation 1976; 53(2): 293-302.

25. Aurigemma GP, Silver KH, Priest MA, Gaasch WH. Geometric changes allow normal ejection fraction despite depressed myocardial shortening in hypertensive left ventricular hypertrophy. J Am Coll Cardiol 1995; 26(1): 195-202.

26. Dong SJ, Hees PS, Huang WM, Buffer SA, Jr., Weiss JL, Shapiro EP. Independent effects of preload, afterload, and contractility on left ventricular torsion. Am J Physiol 1999; 277(3 Pt 2): H1053-H1060. 
27. Jain U, Laflamme CJ, Aggarwal A, Ramsay JG, Comunale ME, Ghoshal S, Ngo L, Ziola K, Hollenberg M, Mangano DT. Electrocardiographic and hemodynamic changes and their association with myocardial infarction during coronary artery bypass surgery. A multicenter study. Multicenter Study of Perioperative Ischemia (McSPI) Research Group. Anesthesiology 1997; 86(3): 576-591.

28. Rankin JS, McHale PA, Arentzen CE, Ling D, Greenfield JC, Jr., Anderson RW. The three-dimensional dynamic geometry of the left ventricle in the conscious dog. Circ Res 1976; 39(3): 304-313.

29. Sengupta PP, Krishnamoorthy VK, Korinek J, Narula J, Vannan MA, Lester SJ, Tajik JA, Seward JB, Khandheria BK, Belohlavek M. Left ventricular form and function revisited: applied translational science to cardiovascular ultrasound imaging. J Am Soc Echocardiogr 2007; 20(5): 539-551.

30. Torrent-Guasp F, Kocica MJ, Corno AF, Komeda M, Carreras-Costa F, Flotats A, Cosin-Aguillar J, Wen H. Towards new understanding of the heart structure and function. Eur J Cardiothorac Surg 2005; 27(2): 191-201.

31. Ross J, Jr., Sonnenblick EH, Covell JW, Kaiser G, Spiro D. The architecture of the heart in systole and diastole. Technique of rapid fixation and analysis of left ventricular geometry. Circ Res 1967; 21(4): 409-421.

32. Sengupta PP, Khandheria BK, Korinek J, Wang J, Belohlavek M. Biphasic tissue Doppler waveforms during isovolumic phases are associated with asynchronous deformation of subendocardial and subepicardial layers. J Appl Physiol 2005; 99(3): 1104-1111.

33. Sengupta PP, Khandheria BK, Korinek J, Wang J, Jahangir A, Seward JB, Belohlavek M. Apex-to-base dispersion in regional timing of left ventricular shortening and lengthening. J Am Coll Cardiol 2006; 47(1): 163-172.

34. Gibbons Kroeker CA, Tyberg JV, Beyar R. Effects of load manipulations, heart rate, and contractility on left ventricular apical rotation. An experimental study in anesthetized dogs. Circulation 1995; 92(1): 130-141.

35. Helle-Valle T, Edvardsen T, Crosby J, Amundsen B, Smith HJ, Rosen BD, Torp H, Stoylen A, Ihlen H, Smiseth O. New non-invasive method for assessment of LV rotation - Speckle tracking echocardiography. Circulation 2004; 110(17): 674-674.

36. Rademakers FE, Rogers WJ, Guier WH, Hutchins GM, Siu CO, Weisfeldt ML, Weiss JL, Shapiro EP. Relation of regional cross-fiber shortening to wall thickening in the intact heart. Three-dimensional strain analysis by NMR tagging. Circulation 1994; 89(3): 1174-1182.

37. Ito BR. Gradual onset of myocardial ischemia results in reduced myocardial infarction. Association with reduced contractile function and metabolic downregulation. Circulation 1995; 91(7): 2058-2070.

38. Derumeaux G, Ovize M, Loufoua J, ndre-Fouet X, Minaire Y, Cribier A, Letac B. Doppler tissue imaging quantitates regional wall motion during myocardial ischemia and reperfusion. Circulation 1998; 97(19): 1970-1977. 
39. Comunale ME, Body SC, Ley C, Koch C, Roach G, Mathew JP, Herskowitz A, Mangano DT. The concordance of intraoperative left ventricular wall-motion abnormalities and electrocardiographic S-T segment changes: association with outcome after coronary revascularization. Multicenter Study of Perioperative Ischemia (McSPI) Research Group. Anesthesiology 1998; 88(4): 945-954.

40. Skulstad H, Andersen K, Edvardsen T, Rein KA, Tonnessen TI, Hol PK, Fosse E, Ihlen H. Detection of ischemia and new insight into left ventricular physiology by strain Doppler and tissue velocity imaging: assessment during coronary bypass operation of the beating heart. J Am Soc Echocardiogr 2004; 17(12): 1225-1233.

41. Edvardsen T, Urheim S, Skulstad H, Steine K, Ihlen H, Smiseth OA. Quantification of left ventricular systolic function by tissue Doppler echocardiography - Added value of measuring pre- and postejection velocities in ischemic myocardium. Circulation 2002; 105(17): 2071-2077.

42. Lim MJ, Karolle BL, Wood JC, Buda AJ. Ischemic expansion during acute myocardial infarction and reversal by coronary reperfusion. Am Heart $J$ 1992; 123(6): 1456-1463.

43. Urheim S, Edvardsen T, Torp H, Angelsen B, Smiseth OA. Myocardial strain by Doppler echocardiography - Validation of a new method to quantify regional myocardial function. Circulation 2000; 102(10): 1158-1164.

44. Urheim S, Edvardsen T, Skulstad H, Ihlen H, Smiseth OA. Assessment of myocardial pressure strain relations by strain rate imaging. Circulation 1999; 100(18): 776-776.

45. Casaburi R. Activity monitoring in assessing activities of daily living. COPD 2007; 4(3): 251-255.

46. Rickards AF, Bombardini T, Corbucci G, Plicchi G. An implantable intracardiac accelerometer for monitoring myocardial contractility. The Multicenter PEA Study Group. Pacing Clin Electrophysiol 1996; $19(12$ Pt 1): 2066-2071.

47. Theres HP, Kaiser DR, Nelson SD, Glos M, Leuthold T, Baumann G, Sowelam S, Sheldon TJ, Stylos L. Detection of acute myocardial ischemia during percutaneous transluminal coronary angioplasty by endocardial acceleration. Pacing Clin Electrophysiol 2004; 27(5): 621-625.

48. Ishimitsu T, Smith D, Berko B, Craige E. Origin of the third heart sound: comparison of ventricular wall dynamics in hyperdynamic and hypodynamic types. $J$ Am Coll Cardiol 1985; 5 (2 Pt 1): 268-272.

49. Wood JC, Buda AJ, Barry DT. Time-frequency transforms: a new approach to first heart sound frequency dynamics. IEEE Trans Biomed Eng 1992; 39(7): 730-740.

50. Wood JC, Festen MP, Lim MJ, Buda AJ, Barry DT. Regional effects of myocardial ischemia on epicardially recorded canine first heart sounds. J Appl Physiol 1994; 76(1): 291-302.

51. Clark JD, Gebhart GF, Gonder JC, Keeling ME, Kohn DF. Special Report: The 1996 Guide for the Care and Use of Laboratory Animals. ILAR J 1997; 38(1): 41-48. 
52. Imenes K, Aasmundtveit K, Husa EM, Hogetveit JO, Halvorsen S, Elle OJ, Mirtaheri P, Fosse E, Hoff L. Assembly and packaging of a three-axis micro accelerometer used for detection of heart infarction. Biomed Microdevices 2007;

53. Bergsland J, Lingaas PS, Skulstad H, Hol PK, Halvorsen PS, Andersen R, Smastuen M, Lundblad R, Svennevig J, Andersen K, Fosse E. Intracoronary shunt prevents ischemia in off-pump coronary artery bypass surgery. Ann Thorac Surg 2009; 87(1): 54-60.

54. Takayama M, Norris RM, Brown MA, Armiger LC, Rivers JT, White HD. Postsystolic shortening of acutely ischemic canine myocardium predicts early and late recovery of function after coronary artery reperfusion. Circulation 1988; 78(4): 9941007.

55. Aase SA, Stoylen A, Ingul CB, Frigstad S, Torp H. Automatic timing of aortic valve closure in apical tissue Doppler images. Ultrasound Med Biol 2006; 32(1): 19-27.

56. Ozawa Y, Smith D, Craige E. Origin of the third heart sound. I. Studies in dogs. Circulation 1983; 67(2): 393-398.

57. Ozawa Y, Smith D, Craige E. Origin of the third heart sound. II. Studies in human subjects. Circulation 1983; 67(2): 399-404.

58. Pauliks LB, Vogel M, Madler CF, Williams RI, Payne N, Redington AN, Fraser AG. Regional response of myocardial acceleration during isovolumic contraction during dobutamine stress echocardiography: a color tissue Doppler study and comparison with angiocardiographic findings. Echocardiography 2005; 22(10): 797-808.

59. Skulstad H, Urheim S, Edvardsen T, Andersen K, Lyseggen E, Vartdal T, Ihlen H, Smiseth OA. Grading of myocardial dysfunction by tissue Doppler echocardiography: a comparison between velocity, displacement, and strain imaging in acute ischemia. $J$ Am Coll Cardiol 2006; 47(8): 1672-1682.

60. Vogel M, Schmidt MR, Kristiansen SB, Cheung M, White PA, Sorensen K, Redington AN. Validation of myocardial acceleration during isovolumic contraction as a novel noninvasive index of right ventricular contractility: comparison with ventricular pressure-volume relations in an animal model. Circulation 2002; 105(14): 1693-1699.

61. Vogel M, Cheung MM, Li J, Kristiansen SB, Schmidt MR, White PA, Sorensen K, Redington AN. Noninvasive assessment of left ventricular force-frequency relationships using tissue Doppler-derived isovolumic acceleration: validation in an animal model. Circulation 2003; 107(12): 1647-1652.

62. Lyseggen E, Rabben SI, Skulstad H, Urheim S, Risoe C, Smiseth OA. Myocardial acceleration during isovolumic contraction: relationship to contractility. Circulation 2005; 111(11): 1362-1369.

63. Blick EF, Sabbah HN, Stein PD. One-dimensional model of diastolic semilunar valve vibrations productive of heart sounds. J Biomech 1979; 12(3): 223-227.

64. Ghista DN, Sandler H, Vayo WH. Elastic modulus of the human intact left ventricle-determination and physiological interpretation. Med Biol Eng 1975; 13(2): 151-161. 
65. Mazumdar J, Woodard-Knight D. A mathematical study of semilunar valve vibration. J Biomech 1984; 17(8): 639-641.

66. Hoff L, Elle OJ, Grimnes MJ, Halvorsen S, Alker HJ, Fosse E. Measurements of heart motion using accelerometers. Conf Proc IEEE Eng Med Biol Soc 2004; 32049-2051.

67. Forrester JS, Tyberg JV, Wyatt HL, Goldner S, Parmely WW, Swan HJ. Pressurelength loop: a new method for simultaneous measurement of segmental and total cardiac function. $J$ Appl Physiol 1974; 37(5): 771-775.

68. D'Ambra MN, Magrassi P, Lowenstein E, Kyo S, Austen WG, Buckley MJ, LaRaia PJ. Myocardial temperature variation: effect on regional function and coronary flow in dogs. Am J Physiol 1987; 252(2 Pt 2): H448-H455.

69. Skulstad H, Edvardsen T, Urheim S, Rabben SI, Stugaard M, Lyseggen E, Ihlen H, Smiseth OA. Postsystolic shortening in ischemic myocardium: active contraction or passive recoil? Circulation 2002; 106(6): 718-724.

70. Bovendeerd PH, Arts T, Huyghe JM, van Campen DH, Reneman RS. Dependence of local left ventricular wall mechanics on myocardial fiber orientation: a model study. $J$ Biomech 1992; 25(10): 1129-1140.

71. Edvardsen T, Skulstad H, Aakhus S, Urheim S, Ihlen H. Regional myocardial systolic function during acute myocardial ischemia assessed by strain Doppler echocardiography. J Am Coll Cardiol 2001; 37(3): 726-730.

72. Gjesdal O, Hopp E, Vartdal T, Lunde K, Helle-Valle T, Aakhus S, Smith HJ, Ihlen H, Edvardsen T. Global longitudinal strain measured by two-dimensional speckle tracking echocardiography is closely related to myocardial infarct size in chronic ischaemic heart disease. Clin Sci (Lond) 2007; 113(6): 287-296.

73. Amundsen BH, Helle-Valle T, Edvardsen T, Torp H, Crosby J, Lyseggen E, Stoylen A, Ihlen H, Lima JA, Smiseth OA, Slordahl SA. Noninvasive myocardial strain measurement by speckle tracking echocardiography: validation against sonomicrometry and tagged magnetic resonance imaging. J Am Coll Cardiol 2006; 47(4): 789-793.

74. Gleason WL, BRAUNWALD E. Studies on the first derivative of the ventricular pressure pulse in man. J Clin Invest 1962; 4180-91.

75. Mason DT, BRAUNWALD E, Covell JW, Sonnenblick EH, Ross J, Jr. Assessment of cardiac contractility. The relation between the rate of pressure rise and ventricular pressure during isovolumic systole. Circulation 1971; 44(1): 47-58.

76. Pinsky MR. Functional hemodynamic monitoring. Intensive Care Med 2002; 28(4): 386-388.

77. Buhre W, Weyland A, Kazmaier S, Hanekop GG, Baryalei MM, Sydow M, Sonntag $\mathrm{H}$. Comparison of cardiac output assessed by pulse-contour analysis and thermodilution in patients undergoing minimally invasive direct coronary artery bypass grafting. J Cardiothorac Vasc Anesth 1999; 13(4): 437-440. 
78. Felbinger TW, Reuter DA, Eltzschig HK, Moerstedt K, Goedje O, Goetz AE. Comparison of pulmonary arterial thermodilution and arterial pulse contour analysis: Evaluation of a new algorithm. Journal of Clinical Anesthesia 2002; 14(4): 296-301.

79. Godje O, Thiel C, Lamm P, Reichenspurner H, Schmitz C, Schutz A, Reichart B. Less invasive, continuous hemodynamic monitoring during minimally invasive coronary surgery. Ann Thorac Surg 1999; 68(4): 1532-1536.

80. Bland JM, Altman DG. Statistical methods for assessing agreement between two methods of clinical measurement. Lancet 1986; 1(8476): 307-310.

81. Buckberg G, Hoffman JI, Mahajan A, Saleh S, Coghlan C. Cardiac mechanics revisited: the relationship of cardiac architecture to ventricular function. Circulation 2008; 118(24): 2571-2587.

82. Peverill RE, Gelman JS, Mottram PM, Moir S, Jankelowitz C, Bain JL, Donelan L. Factors associated with mitral annular systolic and diastolic velocities in healthy adults. J Am Soc Echocardiogr 2004; 17(11): 1146-1154.

83. Heimdal A, Stoylen A, Torp H, Skjaerpe T. Real-time strain rate imaging of the left ventricle by ultrasound. J Am Soc Echocardiogr 1998; 11(11): 1013-1019.

84. Gibbons Kroeker CA, Ter Keurs HE, Knudtson ML, Tyberg JV, Beyar R. An optical device to measure the dynamics of apex rotation of the left ventricle. Am J Physiol 1993; 265(4 Pt 2): H1444-H1449.

85. Kirn B, Starc V. The video technique developed for measuring epicardial strains on guinea pig's left ventricle. J Biomech 2005; 38(1): 169-173.

86. Kirn B, Starc V. Contraction wave in axial direction in free wall of guinea pig left ventricle. Am J Physiol Heart Circ Physiol 2004; 287(2): H755-H759.

87. Arts T, Reneman RS. Measurement of deformation of canine epicardium in vivo during cardiac cycle. Am J Physiol 1980; 239(3): H432-H437.

88. Prinzen FW, Arts T, Van D, V, Reneman RS. Fiber shortening in the inner layers of the left ventricular wall as assessed from epicardial deformation during normoxia and ischemia. J Biomech 1984; 17(10): 801-811.

89. Marcelli E, Plicchi G, Cercenelli L, Bortolami F. First experimental evaluation of cardiac apex rotation with an epicardial coriolis force sensor. ASAIO J 2005; 51(6): 696-701.

90. Olsen CO, Rankin JS, Arentzen CE, Ring WS, McHale PA, Anderson RW. The deformational characteristics of the left ventricle in the conscious dog. Circ Res 1981; 49(4): 843-855.

91. Espinoza A, Halvorsen PS, Hoff L, Skulstad H, Fosse E, Ihlen H, Edvardsen T. Detecting myocardial ischaemia using miniature ultrasonic transducers--a feasibility study in a porcine model. Eur J Cardiothorac Surg 2010; 37(1): 119-126. 
92. Remme EW, Hoff L, Halvorsen PS, Naerum E, Skulstad H, Fleischer LA, Elle OJ, Fosse E. Validation of cardiac accelerometer sensor measurements. Physiol Meas 2009; 30(12): 1429-1444.

93. Schwartz GG, Schaefer S, Meyerhoff DJ, Gober J, Fochler P, Massie B, Weiner MW. Dynamic relation between myocardial contractility and energy metabolism during and following brief coronary occlusion in the pig. Circ Res 1990; 67(2): 490-500.

94. Jennings RB, Steenbergen C, Jr., Reimer KA. Myocardial ischemia and reperfusion. Monogr Pathol 1995; 3747-80.

95. Jamal F, Strotmann J, Weidemann F, Kukulski T, D'hooge J, Bijnens B, Van de WF, De S, I, Sutherland GR. Noninvasive quantification of the contractile reserve of stunned myocardium by ultrasonic strain rate and strain. Circulation 2001; 104(9): 1059-1065. 
9. Papers 



\title{
Early recognition of regional cardiac ischemia using a 3-axis accelerometer sensor
}

\author{
Ole Jakob Elle ${ }^{1}$, Steinar Halvorsen ${ }^{1}$, Martin Gunnar Gulbrandsen ${ }^{1}$, \\ Lars Aurdal $^{1}$, Andre Bakken ${ }^{2}$, Eigil Samset ${ }^{1}$, Harald Dugstad ${ }^{1}$ \\ and Erik Fosse ${ }^{1}$ \\ ${ }^{1}$ The Interventional Centre, Rikshospitalet University Hospital, Oslo, Norway \\ ${ }^{2}$ Department of Clinical Engineering, Rikshospitalet University Hospital, Oslo, Norway
}

Received 22 December 2004, accepted for publication 8 March 2005

Published 15 April 2005

Online at stacks.iop.org/PM/26/429

\begin{abstract}
Perioperative mortality in coronary artery bypass grafting is usually caused by reduced left ventricular function due to regional myocardial ischemia or infarction. Post-operative graft occlusion is a well-known problem in coronary surgery. A sensitive tool to detect graft occlusion and monitor myocardial function may give the opportunity to revise malfunctioning grafts before departure from the hospital. This paper describes how a new method can detect cardiac ischemia using a 3-axis piezoelectric accelerometer. In three anesthetized pigs, a 3-axis piezoelectric accelerometer was sutured on the lateral free wall of the left ventricle. The left anterior descending (LAD) was occluded for different time periods and the accelerometer data were sampled with a PC. Short-time Fourier transform was calculated based on the accelerometer time series. The results were visualized using a $2 \mathrm{D}$ color-coded time-frequency plot. In the area of occlusion, a change to stronger power of higher harmonics was observed. Consequently, a difference value between the instant frequency pattern and a reference frequency pattern showed a rise in absolute value during the occlusion period. The preliminary results indicate that early recognition of regional cardiac ischemia is possible by analyzing accelerometer data acquired from the three animal trials using the prototype 3 -axis accelerometer sensor.
\end{abstract}

Keywords: biomedical sensor, myocardial ischemia, coronary bypass, accelerometer, fast Fourier transform (FFT)

(Some figures in this article are in colour only in the electronic version)

\section{Introduction}

Post-operative graft occlusion is a well-known problem in coronary surgery; 2-6\% of arterial and $10-20 \%$ of venous grafts are occluded after 1 year (Hol et al 2004). A number of 
occlusions occur immediately after surgery. A sensitive tool to detect graft occlusion and monitor myocardial function may give the opportunity to revise malfunctioning grafts before departure from the hospital. We have previously reported that $4 \%$ of grafts were malfunctioning on angiography obtained in the operation theatre immediately after closure of the wound (Hol et al 2004).

Myocardial ischemia after coronary artery bypass grafting (CABG) can be detected by ECG changes, and eventually by reduction in the cardiac pump function as measured by invasive hemodynamic monitoring of the heart. These findings, however, are not consistent due to poor sensitivity and specificity of both methods (Jain et al 1997, Gianrossi et al 1989).

Angiography is commonly used to identify stenotic and occluded coronary arteries. Echocardiography is used in the diagnostics of cardiac contractility, hemodynamic performance and valve function. Regional reduced contractility or paradox movement may indicate which coronary artery is occluded. Strain and tissue velocity are new echocardiographic methods for estimation of the viability and the contractility of the myocardium (Edvardsen et al 2001). However, the method is user-dependent and is unsuitable for continuous post-operative monitoring due to the need for a cardiologist holding the probe and analyzing the visual output.

Accelerometer sensors have been used in implantable cardiac pacemakers, such as the pacing leads from Guidant (Guidant Corporation, Indianapolis, USA, www.guidant.com), to sense abnormal heart activity. Academic papers have also addressed the idea of using accelerometer sensors to sense different aspects of abnormal heart activity. Some of these have detected motion changes at the surface of the ventricle by the use of a surface mounted single-axis accelerometer sensor (Ishimitsu et al 1985, Ozawa et al 1983a, 1983b, Wood et al $1992,1994)$. These papers refer to work where the accelerometer data were used with the purpose to provide more knowledge about the mechanics in the generation of the heart sound (as detected by stethoscope) in the different phases of a heart beat cyclus. The analogy between acceleration and sound is easily understood if the heart surface is considered as the loudspeaker membrane and the accelerometer as the microphone picking up the sound changes. A recent publication from Theres et al (2004) addresses detection of myocardial ischemia during percutaneous transluminal coronary angioplasty (PCI) by endocardial acceleration measured by a $1 \mathrm{D}$ accelerometer.

This animal study addresses the development and performance of a prototype 3-axis accelerometer sensor in detecting cardiac ischemia. The accelerometer sensor is based on two commercially available 2-axis sensors mounted perpendicular to each other.

The aim of the present work was to establish whether a 3-axis accelerometer may detect coronary occlusion by analysis of the accelerometer pattern with reasonable sensitivity.

\section{Materials and methods}

\subsection{Prototype development-3D accelerometer}

Two 2-axis accelerometers (ADXL-202) from Analog Devices (Analog Devices Inc, Norwood, MA, USA) were mounted perpendicular to each other in order to make a 3-axis acquisition of the acceleration data. Table 1 shows the specifications for this capacitive accelerometer.

The accelerometer sensors can provide digital or analog output signals proportional to the acceleration. The sensors were tuned according to the instructions from Analog Devices (www.analog.com). An operation amplifier was used as a buffering of the signals to make them more robust to the surrounding noise, especially $50 \mathrm{~Hz}$ from the net. The electronic 


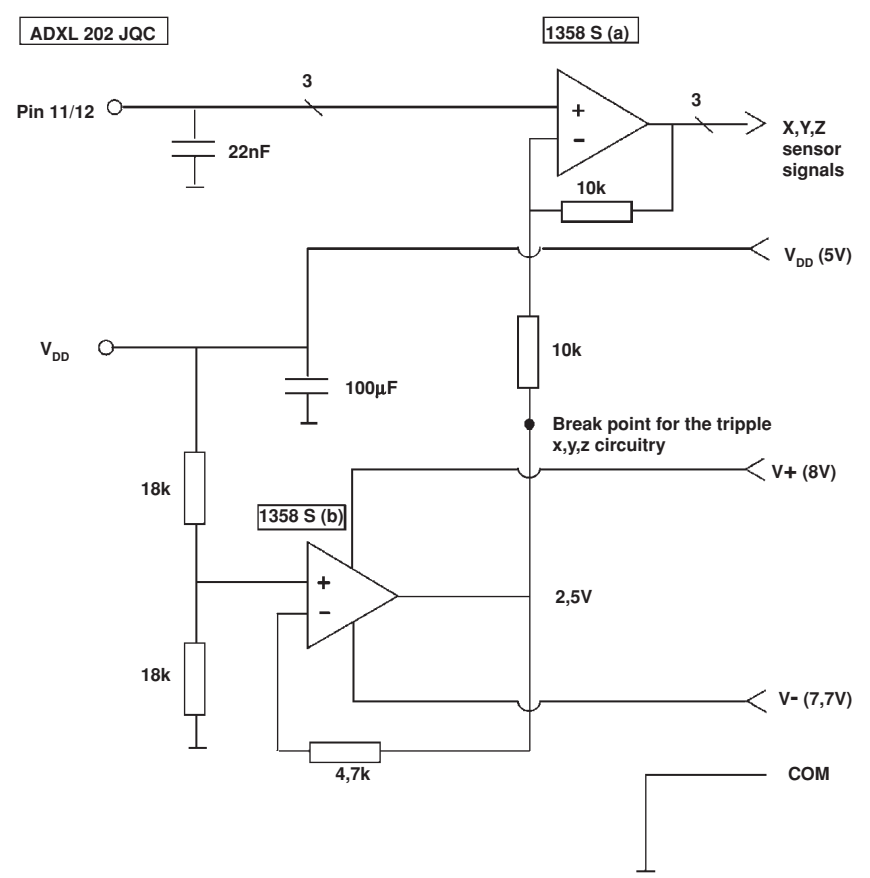

Figure 1. Electronic circuitry of prototype 3D-accelerometer sensor.

Table 1. Specification on the analog output pins for the capacitive accelerometer ADXL-202 from Analog Devices Inc.

\begin{tabular}{ll}
\hline Property & Value \\
\hline Principal & Capacitive acceleration sensor \\
Axes & 2-axis \\
Range & $\pm 2 \mathrm{~g}$ \\
Sensitivity (3 V supply voltage) & Typ. $167 \mathrm{mV} \mathrm{g}^{-1}$ \\
Sensitivity (5 V supply voltage) & Typ. $312 \mathrm{mV} \mathrm{g}^{-1}$ \\
Resolution & $2 \mathrm{mg} \mathrm{at} 60 \mathrm{~Hz}$ \\
Resonant frequency & $10 \mathrm{kHz}$ \\
3 dB bandwidth & $6 \mathrm{kHz}$ \\
Power & $<0.6 \mathrm{~mA}$ \\
Size & $5 \times 5 \times 2 \mathrm{~mm}^{3}$ \\
Supply voltage & $3-5.25 \mathrm{~V}$ \\
Shock survival & $1000 \mathrm{~g}$ \\
Measures & Dynamic (vibrations), static (gravity) \\
\hline
\end{tabular}

circuitry is shown in figure 1 . The electronics together with the two connected accelerometer sensors were put into a polymer box and sealed. Small holes were made in the base plate to apply the sutures when attached to the epicardium. 
Table 2. Timetable for animal experiments. The numbers in brackets show the part of the time series of animal 1 used for spectrograms and FFT Euclidian distance plots (difference value) in figure 5 .

\begin{tabular}{llll}
\hline & Startlog $(a \mathrm{~s})$ & Occlusion time $(b \mathrm{~s})$ & Stoplog $(c \mathrm{~s})$ \\
\hline Animal 1 & $530(80)$ & $370(170)$ & - \\
Animal 2 & 80 & 85 & 35 \\
Animal 3 & 30 & 128 & 60 \\
\hline
\end{tabular}

\subsection{Animal protocol}

The prototype 3-axis accelerometer was tested in a porcine model. In a protocol approved by the institutional Animal Care and Use Committee, three pigs of either sex (mean weight $52.4 \mathrm{~kg} \pm 3.1 \mathrm{SD}$ ) underwent surgery.

2.2.1. Animal preparation. The pigs were kept fasting overnight with free access to water. The animals were sedated with ketamine $20 \mathrm{mg} \mathrm{kg}^{-1}$ intramuscularly and azaperone $2 \mathrm{mg} \mathrm{kg}^{-1}$ preoperatively. Anesthesia was induced with intravenous pentobarbital titrated until no reaction to skin incision $\left(3-6 \mathrm{mg} \mathrm{kg}^{-1}\right)$. Anesthesia was maintained with constant infusion of morphine $20-40 \mathrm{mg} \mathrm{h}^{-1}$ and inhalation of isoflurane $0.5-1.5 \%$ titrated according to autonomic responses to surgery. Immediately after induction of anesthesia, a tracheotomy through a neck midline incision was performed and the pigs were ventilated with tidal volume and rate adjusted to keep $\mathrm{PaCO}_{2}$ close to 40 Torr $(5.3 \mathrm{kPa})$. $\mathrm{FiO}_{2}$ was kept sufficiently high to ensure an arterial $\mathrm{PO}_{2}>100$ Torr $(13.3 \mathrm{kPa})$. The right carotid artery and right internal jugular vein were isolated and cannulated. A single-lumen catheter was advanced into the right internal jugular vein for continuous infusion of isotonic crystalloid solution (Ringer's acetate). For sampling of arterial blood specimens and continuous arterial pressure monitoring, a $5 \mathrm{Fr}$ catheter was advanced into the right carotid artery. An ECG was monitored from a limb lead.

After a median sternotomy, the pericardium was split from apex to base, and a $3 \mathrm{~mm}$ vascular occluder was positioned around left anterior descending (LAD) distal to the first branch ready to occlude according to the protocol. A 3D accelerometer sensor was sutured on the left ventricle free wall in the region of blood supply of the LAD, approximately $2 \mathrm{~cm}$ proximal to apex. The animals were allowed to stabilize for $30 \mathrm{~min}$ to achieve a steady-state condition.

\subsection{Data acquisition}

The DAQ 1200 Data Acquisition Card for PCMCIA bus (National Instruments Inc, Austin, TX, USA) together with a data logger for analog readings programed in Lab View (National Instruments Inc, Austin, TX, USA) was used to log the accelerometer data.

The investigation was performed by totally occluding LAD distal to the first diagonal branch and reperfused according to the protocol (table 2).

1. Occluded left anterior descending artery after $a$ seconds.

2. Then opened LAD for reperfusion of the heart after $b$ seconds.

3. Finally, stopped $\log$ after $c$ seconds.

The accelerations along the three orthogonal axes were logged to a PC with a sampling frequency found to be above the Nyquist limit. 
A : Before occlusion

(G)

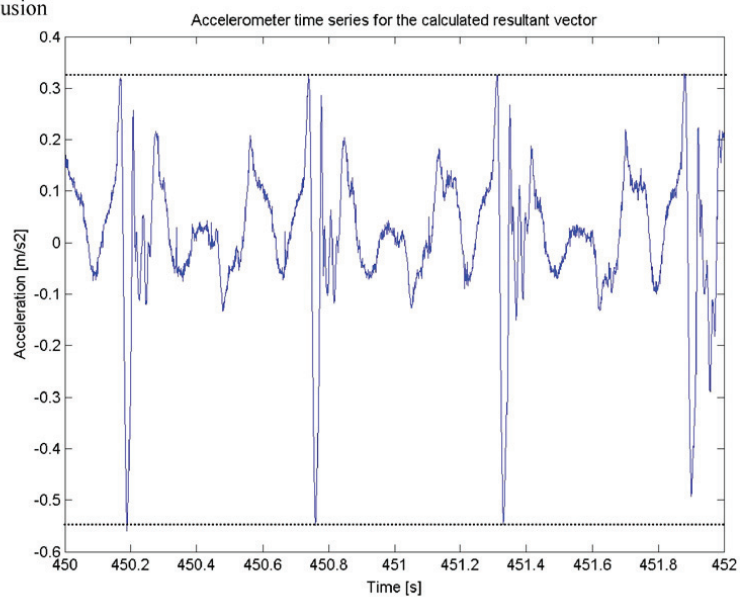

B : After 130 seconds of occlusion

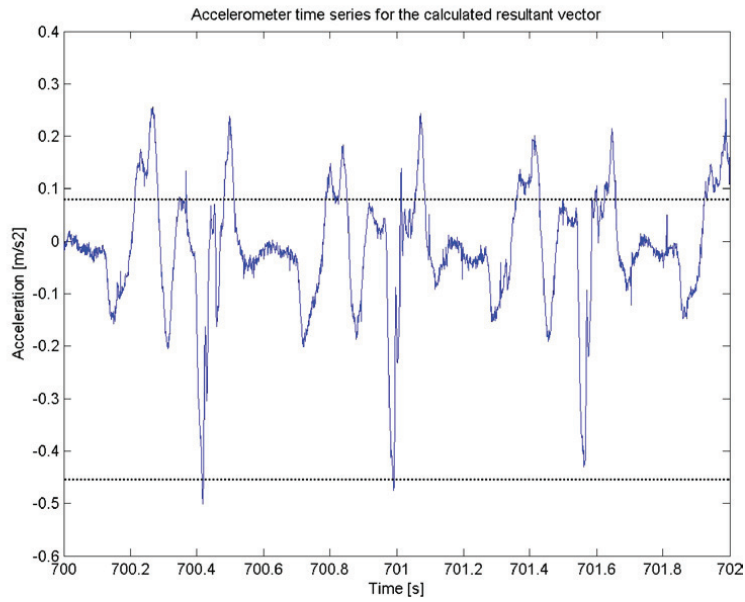

Figure 2. Resultant acceleration raw-data vector of animal 1 before occlusion (upper) and after occlusion (lower).

\subsection{Signal processing}

The signal processing for the detection of ischemia was carried out in MatLab (The MathWorks Inc, MA, USA). The acquired accelerometer signals contained a significant offset due to gravitation. This was removed by subtracting a moving average.

The data from each of the three orthogonal axes were analyzed separately. Also, the vector length of the measured acceleration was investigated as a parameter for analysis (figure 2). 


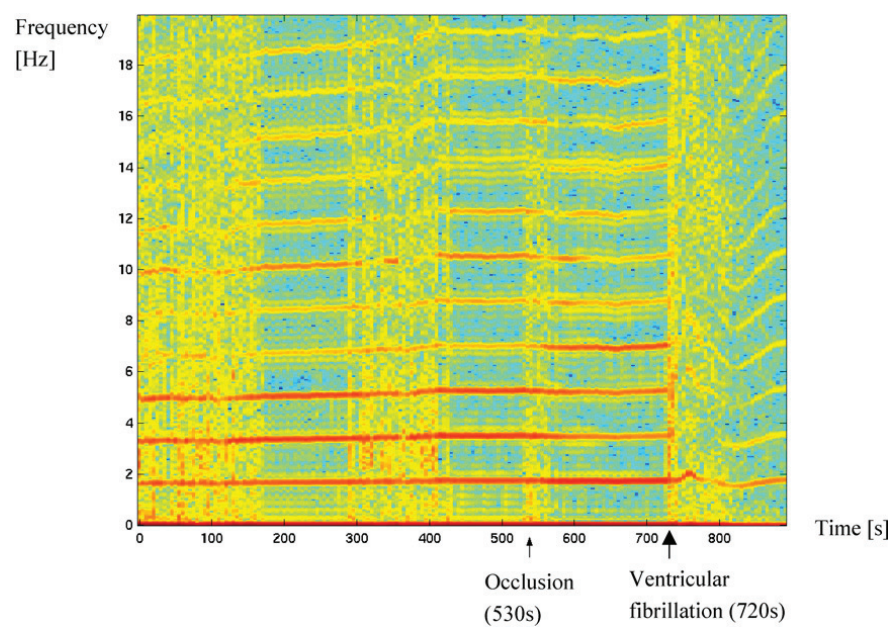

Figure 3. Spectrogram of acceleration signal from animal 1.

Let $a_{i}$ denote the length of the acceleration vector $\left(a_{x i}, a_{y i}, a_{z i}\right)$ at time step $i$. Figure 3 shows the spectrogram generated for this time series resulting from a short-time fast Fourier transform (FFT) with a subvector $\left(a_{n \rightarrow n+1}\right)$ of length $(l)$ sliding from start to end of the time series. The color of each point in the diagram indicates the power of a given frequency component (2: axis) in the time series at a given time (1: axis). Thus, each vertical line shows the approximated short-term frequency distribution at any given time. The scale goes from blue as the weakest via green and yellow to red as the strongest frequency.

A fast Fourier transform with a sliding window of 512 elements was used to analyze the data corresponding to the sampling frequency that were used in the acquisition of the data. It calculates a vector containing the power of each frequency component in the signal, thus giving the frequency distribution of the signal (power spectrum-PS).

The Euclidian distance calculation (difference value) between the power vector (FFT vector) of the actual sliding signal window $(\mathrm{PS}(n))$ and a reference (baseline) window $\left(\mathrm{PS}_{\text {ref }}\right)$ was performed in order to find a single value that might represent the ischemic state of the heart (equation (1)):

$$
\Delta \text { Power }_{\text {tot }}=\sum_{t=1}^{\text {length of log }} \sqrt{\sum_{n=1}^{512}\left(\operatorname{PS}(n)-\mathrm{PS}_{\mathrm{ref}}\right)^{2}} \text {. }
$$

The MatLab implementation of the difference value algorithm is shown in table 3.

\section{Results}

\subsection{Detection of ischemia}

Figure 2 shows the vector length of the acceleration raw-data in $x, y$ and $z$ from animal 1 before (a) and after (b) the $130 \mathrm{~s}$ LAD occlusion time. The frequency pattern has changed due to ischemia. The early positive peak systolic acceleration (PSA) is reduced from approximately 
Table 3. The MatLab implementation of the difference value algorithm. The $x$-, $y$ - and $z$-accelerations are found in the second, third and fourth columns of the data acquisition file, respectively. A 512 points fast Fourier transform is used in the calculation of both the difference value and the spectrogram. The sample frequency is $250 \mathrm{~Hz}$.

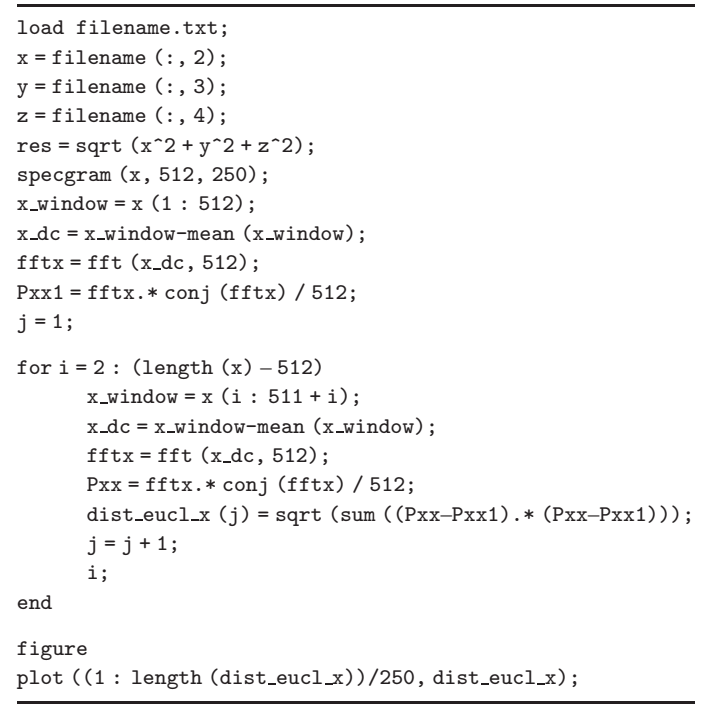

$0.325 \mathrm{~g}$ to approximately $0.075 \mathrm{~g}$, while the negative PSA is reduced from approximately $0.55 \mathrm{~g}$ to approximately $0.45 \mathrm{~g}$ giving a total reduction of peak to peak of about $0.35 \mathrm{~g}$ corresponding to approximately $40 \%$ of baseline peak to peak. Morphological changes during ischemia are observed, but a deeper look into these changes is needed to give more insight into the phenomenon. The choice of signal processing is founded on these findings.

The heartbeat of approximately $1.7 \mathrm{~Hz}$ and its higher order harmonics can be seen as strong horizontal (red) lines (figure 3). The strength of the harmonics of the $1.7 \mathrm{~Hz}$ heartbeat changes after the occlusion. For instance, the fourth harmonic, at approximately $6.5 \mathrm{~Hz}$, is much stronger after the occlusion, whereas the sixth harmonic at roughly $10 \mathrm{~Hz}$ is weaker (figure 3).

Calculation of the total power difference was performed for each of the three axes separately in order to find the direction of myocardial vibration most sensitive to reduced blood supply and ischemia (figure 4). According to the three animals experiments investigated, the transversal direction ( $Y$-direction) gives the most prominent motion change found (figure 4).

Figure 5 shows spectrograms and FFT Euclidian distance plots (difference value) calculated using the accelerometer data transversal to the heart surface ( $Y$-direction) for the three animals investigated. The area of occlusion is marked. Generally, the spectrograms show the power of the different frequencies color coded, with red indicating the strongest power. A rise in power of the higher harmonics is shown for the first animal with a correspondingly steep rise in calculated difference value after $80 \mathrm{~s}$ where the left anterior descending artery was occluded. Looking at the second spectrogram, a significant rise in power of higher harmonics is shown at time $80 \mathrm{~s}$ when occlusion of LAD started. A new change to lower power in the higher harmonics is shown at time $165 \mathrm{~s}$ when the LAD is reperfused. Correspondingly, 

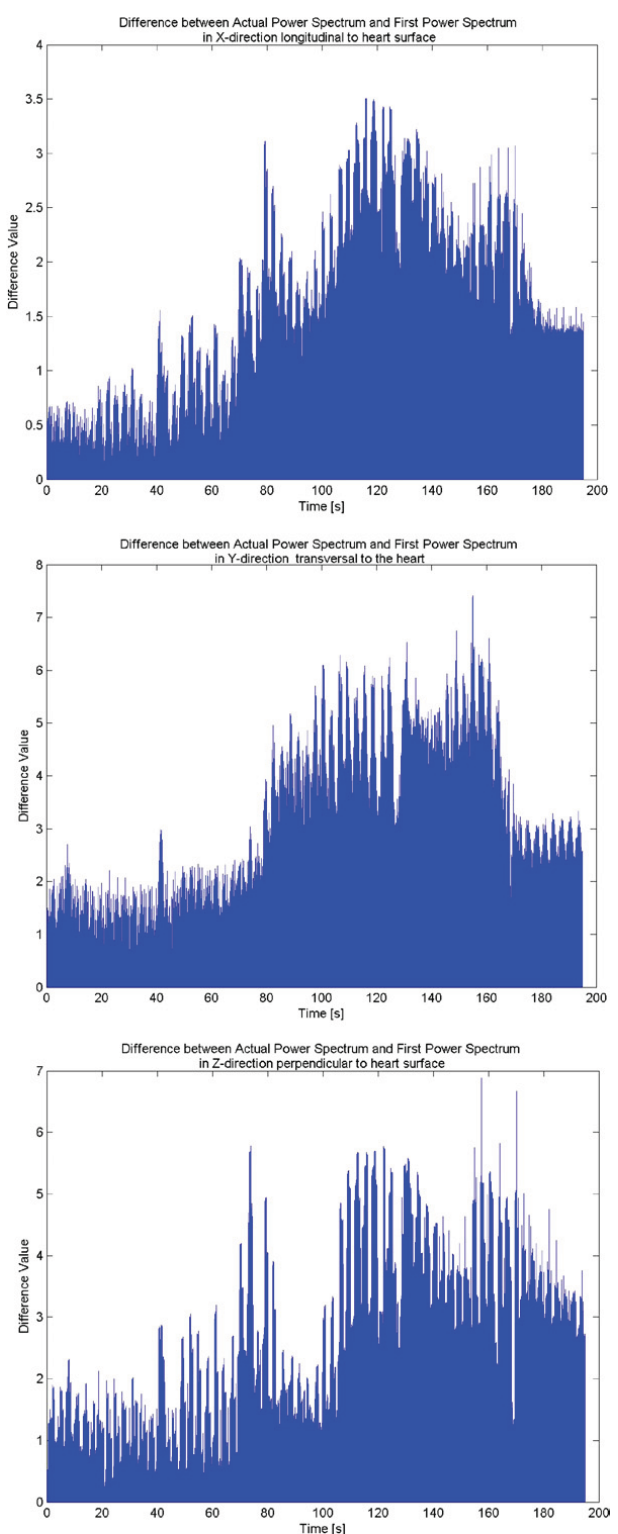

Figure 4. Difference value, calculated as the Euclidian distance between the actual power spectrum and the reference power spectrum in $X$ - (longitudinal), $Y$ - (transversal) and $Z$ - (perpendicular) directions relative to the heart surface. 

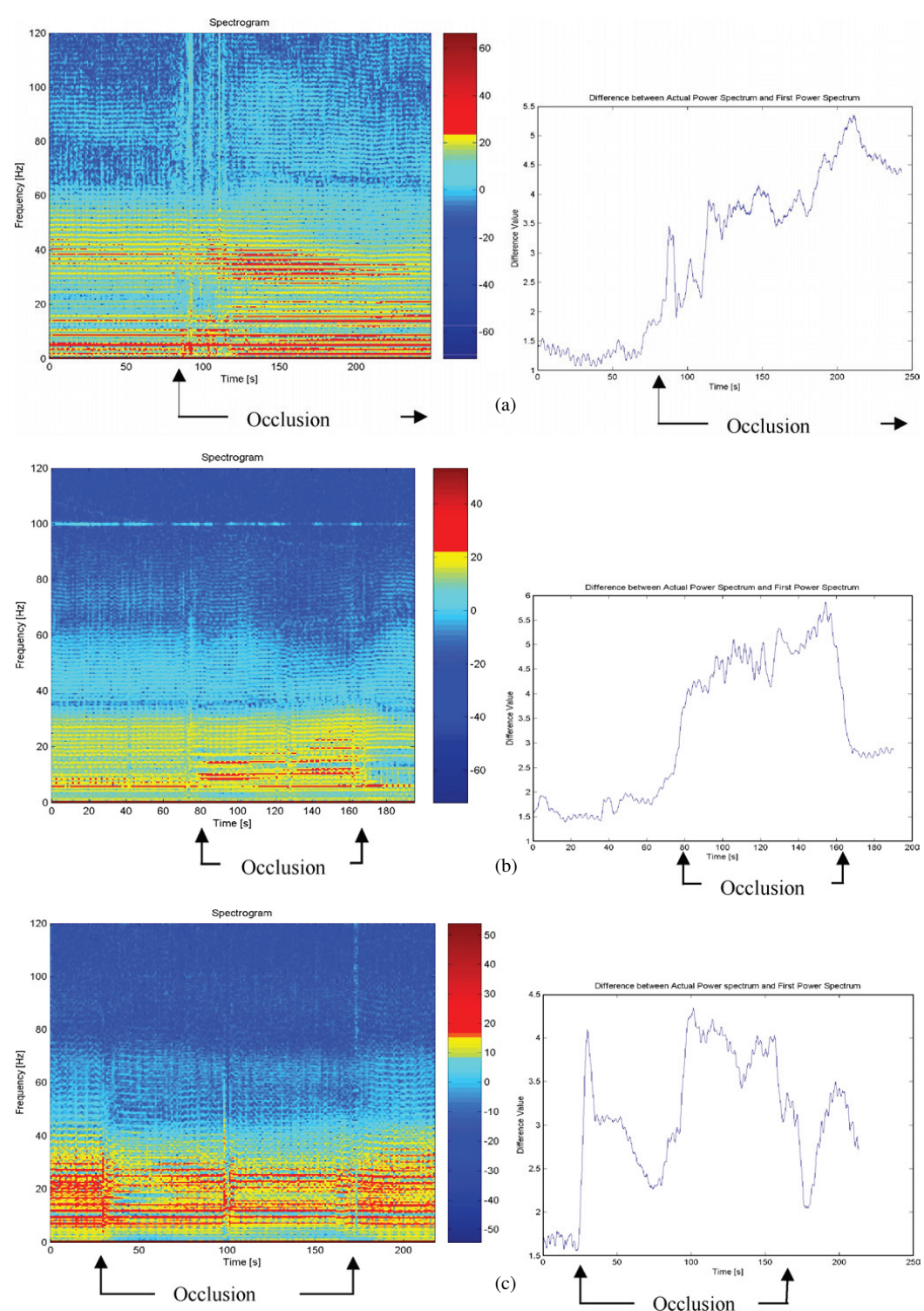

Figure 5. Spectrogram and FFT difference value calculated using the accelerometer data transversal to the heart surface $(Y$-axis) for the three animals investigated. Occlusion and reopening of the LAD are indicated with arrows. 
the Euclidian distance plot shows significant change in the calculated difference value at the point where occlusion and reperfusion took place. The last animal showed changes in the spectrogram and difference value corresponding to occlusion of the LAD between $30 \mathrm{~s}$ and $158 \mathrm{~s}$ though not as clear as the other two.

\section{Discussion}

The preliminary results indicate that early recognition of ischemia is possible through the method described.

The reduction in total peak systolic acceleration during occlusion of the LAD indicates the changes in acceleration due to ischemia (figure 2). This reduction of about $40 \%$ was found after $130 \mathrm{~s}$ of occlusion. A method to detect the early changes in the measured acceleration just after occlusion and during incipient ischemia is proposed using a difference value based on the FFT frequency pattern.

The results also show that the myocardial movements consist of multicomponent periodic signals. The difference between the actual frequency distribution and a reference distribution is calculated as a measure of the cardiac motion pattern. This has an analogy with sound in that the tone level changes according to changes in the frequency. The same tone can have different sound (acoustic footprint) from different instruments according to the different strength of the harmonics it contains. Thus, it is possible to observe the change in frequency pattern from baseline to occlusion and occlusion to reperfusion corresponding to motion changes of the myocardial wall.

The spectrogram in figure 3 seems to give an indication of how to detect a difference in the acceleration signal before and after the occlusion.

One should perhaps not expect the distribution to be completely universal, but there could be universal characteristics of a 'healthy' frequency spectrum. In any case, to warn of ischemia, it is enough to confirm that the frequency distribution is stable in a healthy heart, since then any significant change could trigger a warning. Such a system could be implemented by keeping track of the first harmonics of the heartbeat, which may be accomplished by means of, e.g. a FFT.

Wood et al (1992) described different signal processing and analysis techniques and discussed advantages and disadvantages of these techniques related to the changing dynamic nature of the heart vibrations using the single-axis accelerometer similar to the one used in Ishimitsu et al (1985) and Ozawa et al (1983a, 1983b). They used a one-axis accelerometer from Entran Devices (Entran EGA 125R-30D) oriented with its sensitive axis perpendicular to the heart surface. In Wood et al (1994) knowledge from Wood et al (1992) is used to look at the regional effect of myocardial ischemia related to the first heart sound.

The frequency spectrum changes throughout a heartbeat, and there is a trade-off between frequency resolution and temporal resolution using power spectra since it is basically a time average of a signal's instantaneous frequency spectra (Wood et al 1994). By using bilinear time-frequency transforms where the power spectrum analysis is limited to a sliding window, a better time resolution is acquired sacrificing some of the frequency resolution. The width of the sliding window has to be considered to optimize this method. Other time-frequency transforms avoid this time-bandwidth compromise by using a window function that is the time-reversed signal. The window has a long duration giving good frequency resolution, but the product of the signal against its reversed counterpart simultaneously yields good temporal localization. The Wagner distribution, the Choi-Williams distribution and the binomial transform are of this type. These transforms are discussed in Wood et al $(1992,1994)$ for analysis of the dynamic signal change within a heartbeat investigating the different phases through the heart sound. We 
are more interested in signal changes from heartbeat to heartbeat to detect myocardial ischemia rather than changes within each heartbeat. Therefore, we used the fast Fourier transform with a sliding window containing a couple of heartbeats. Additionally, the fast Fourier transform is widely used in signal analysis and is therefore well documented.

A recent publication from Theres et al (2004) addresses detection of myocardial ischemia during percutaneous coronary intervention by endocardial acceleration. They placed a singleaxis lead-based accelerometer in the RV apex via a femoral approach. The different coronary arteries were occluded using a balloon catheter in the actual artery. A 12-lead surface ECG was continuously monitored. Changes in measured acceleration due to artery occlusion were calculated as peak endocardial acceleration (PEA), time of endocardial acceleration (TEA) and Max Filteres EA. A significant drop of $7 \%$ was found in PEA and Max Filteres EA $(p<0.05)$. TEA did not change significantly.

In this paper, peak systolic acceleration is defined as the maximum acceleration observed on the myocardial surface in systolic contraction. A reduction in peak-to-peak PSA of about $40 \%$ was observed.

The method described in this paper differs from the method described by Theres et al (2004) mainly in two aspects. The first is that the acceleration sensors in this paper are attached to the heart surface during coronary artery bypass grafting to monitor changes in heart movements postoperatively due to occlusion of the coronary arteries. The method described by Theres et al describes a transluminal method that is useful in percutaneus coronary intervention where a blocking of the occluded artery or placement of a stent in the artery is the treatment of the coronary disease. Second, this paper describes a method where a 3-axis accelerometer is used to make the method insensitive to in which orientation the sensor is attached as opposed to Theres' method where the single-axis is placed in a predetermined direction. Valuable information for sensitivity of the method will be lost when the direction of the sensor axis is not aligned with the direction giving the most prominent motion changes. Thus, the method can fail or give less good sensitivity and specificity in detection of myocardial ischemia.

As seen from figure 4, the direction transversal ( $Y$-direction) to the heart surface is the one showing the most clean and prominent change in power due to occlusion and reperfusion of the LAD. The normal to the heart surface (Z-direction) shows the less prominent change, and both the longitudinal ( $X$-direction) and the normal direction are probably affected by disturbances due to the occlusion and reperfusion process of the LAD.

To make the detection method insensitive to the orientation of the accelerometer sensor, the length of the 3-axis accelerometer vector can be used as a resultant vector. Using the resultant from the spatial accelerometer data, user friendliness is provided in that the attachment of the sensor device to the heart surface is independent of the orientation of the sensor.

From the spectrogram of the second experiment that is shown in figure 5, a rise in power of the higher harmonics is observed in the area of occlusion. The rise in calculated difference value confirms this observation. The spectrogram of the third experiment shows a general impairment of the power when occluded. The calculated difference value is squared providing the magnitude of the difference in the frequency distribution.

The calculated difference value of the second experiment shown in figure 5 shows that the magnitude of the value before occlusion of LAD is lower than after reopening of LAD (reperfusion). This phenomenon could have a connection to a preconditioning of the heart, where the change in frequency pattern is prolonged after occlusion.

The performed experiments indicate that the frequency spectrum of the acceleration signal can be used to detect ischemia. Although the present study demonstrated an immediate change in regional wall motion pattern when the coronary vessel was occluded, further studies are needed to establish reproducibility, sensitivity and specificity of the method. 
Given favorable results, continuous wall motion monitoring by a miniaturized accelerometer sensor incorporated into a temporary pacing lead may prove a new clinical tool for coronary surgery patient monitoring.

\section{Conclusion}

The experiments show that accelerometer data can be used to detect regional cardiac ischemia. A rapid change in frequency distribution from the accelerometer data measured occluding the blood flow in the LAD indicates that early recognition of regional myocardial ischemia can be provided by analyzing accelerometer data real-time using fast Fourier transform. The Euclidian distance calculation (difference value) can be used to track changes between reference and actual frequency distribution in time. By proper selection of limit values, a single value can be used to raise an alarm if the limit is exceeded indicating possible ischemia.

In this animal model, the LAD was occluded distal to the first diagonal branch producing significant ischemia. Further study is needed to detect graft occlusion with only partial impaired blood supply.

Disclosure: A patent application has been filed on this concept and the preliminary result (Elle et al 2003).

\section{References}

Edvardsen T, Skulstad H, Aakhus S, Urheim S and Ihlen H 2001 Regional myocardial systolic function during acute myocardial ischemia assessed by strain Doppler echocardiography J. Am. Coll. Cardiol. 37 726-30

Elle O J, Fosse E and Gulbrandsen M G 2003 International Patent Application with International Publication Number WO 03/061473

Gianrossi R, Detrano R, Mulvihill D, Lehmann K, Dubach P, Colombo A, McArthur D and Froelicher V 1989 Exercise-induced ST depression in the diagnosis of coronary disease: a meta-analysis Circulation 80 87-98

Hol P K, Lingaas P S, Lundblad R, Rein K A, Vatne K, Smith H J, Nitter-Hauge S and Fosse E 2004 Intraoperative angiography leads to graft revision in coronary artery bypass surgery Ann. Thor. Surg. 78 502-5

Ishimitsu T, Smith D, Berko B and Craige E 1985 Origin of the third heart sound: comparison of ventricular wall dynamics in hyperdynamic and hypodynamic types $J$. Am. Coll. Cardiol. 5 268-72

Jain U, Laflamme C J, Aggarwal A, Ramsay J G, Comunale M E, Ghoshal S, Ngo L, Ziola K, Hollenberg M and Mangano D T 1997 Electrocardiogrphic and hemodynamic changes and their association with myocardial infarction during coronary artery bypass surgery Anesthesiology 86 576-91

Ozawa Y, Smith D and Craige E 1983a Origin of the third heart sound. I. Studies in dogs Circulation 67 393-8

Ozawa Y, Smith D and Craige E 1983b Origin of the third heart sound. II. Studies in human subjects Circulation 67 399-404

Theres H P, Kaiser D R, Nelson S D, Glos M, Leuthold T, Baumann G, Sowelam S, Sheldon T J and Stylos L 2004 Detection of acute myocardial ischemia during percutaneous transluminal coronary angioplasty by endocardial acceleration Pacing Clin. Electrophysiol. 27621

Wood J C, Buda A J and Barry D T 1992 Time-frequency transforms: a new approach to first heart sound frequency dynamics IEEE. Trans. Biomed. Eng. $39730-40$

Wood J C, Festen M P, Lim M J, Buda A J and Barry D T 1994 Regional effects of myocardial ischemia on epicardially recorded canine first heart sounds J. Appl. Physiol. 76 291-302 


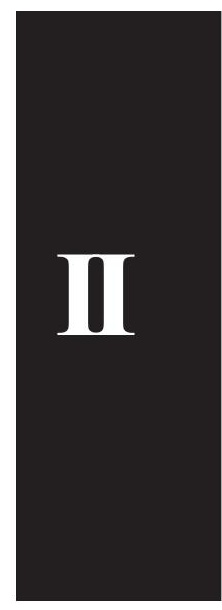





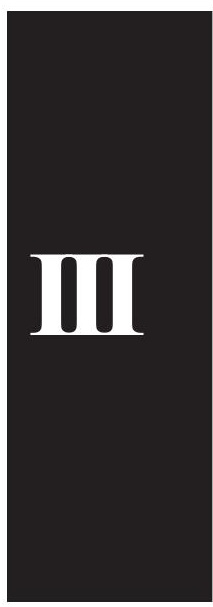





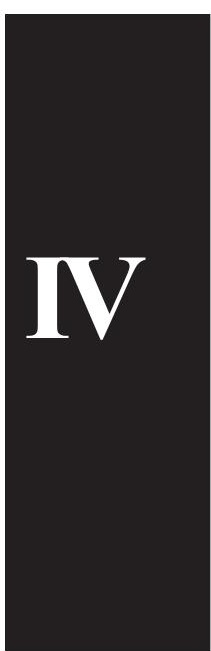


